\title{
Kawasaki Dynamics in Continuum: Micro- and Mesoscopic Descriptions
}

\author{
Christoph Berns • Yuri Kondratiev • Yuri Kozitsky • \\ Oleksandr Kutoviy
}

Received: 31 July 2011 / Revised: 18 August 2013 / Published online: 21 November 2013

(C) The Author(s) 2013. This article is published with open access at Springerlink.com

\begin{abstract}
The dynamics of an infinite system of point particles in $\mathbb{R}^{d}$, which hop and interact with each other, is described at both micro- and mesoscopic levels. The states of the system are probability measures on the space of configurations of particles. For a bounded time interval $[0, T)$, the evolution of states $\mu_{0} \mapsto \mu_{t}$ is shown to hold in a space of sub-Poissonian measures. This result is obtained by: (a) solving equations for correlation functions, which yields the evolution $k_{0} \mapsto k_{t}, t \in[0, T)$, in a scale of Banach spaces; (b) proving that each $k_{t}$ is a correlation function for a unique measure $\mu_{t}$. The mesoscopic theory is based on a Vlasov-type scaling, that yields a mean-field-like approximate description in terms of the particles' density which obeys a kinetic equation. The latter equation is rigorously derived from that for the correlation functions by the scaling procedure. We prove that the kinetic equation has a unique solution $\varrho_{t}, t \in[0,+\infty)$.
\end{abstract}

Keywords Infinite particle system $\cdot$ Microscopic dynamics $\cdot$ Kinetic equation

\author{
C. Berns · Y. Kondratiev · O. Kutoviy \\ Fakultät für Mathematik, Universität Bielefeld, Postfach 100 131, \\ 33501 Bielefeld, Germany \\ e-mail: cberns@math.uni-bielefeld.de \\ Y. Kondratiev \\ e-mail: kondrat@math.uni-bielefeld.de \\ O. Kutoviy \\ e-mail: kutoviy@math.uni-bielefeld.de \\ Y. Kozitsky $(\varangle)$ \\ Instytut Matematyki, Uniwersytet Marii Curie-Skłodowsiej, 20-031 Lublin, Poland \\ e-mail: jkozi@hektor.umcs.lublin.pl \\ O. Kutoviy \\ Department of Mathematics, Massachusetts Institute of Technology, \\ 77 Massachusetts Avenue E18-420, Cambridge, MA 02139, USA \\ e-mail: kutovyi@mit.edu
}




\section{Introduction}

\subsection{The Setup}

In this paper, we study the dynamics of an infinite system of point particles in $\mathbb{R}^{d}$ which hop and interact with each other. The corresponding phase space is the set of configurations

$$
\Gamma=\left\{\gamma \subset \mathbb{R}^{d}:|\gamma \cap K|<\infty \text { for any compact } K \subset \mathbb{R}^{d}\right\},
$$

where $|A|$ denotes the cardinality of a finite set $A$. The set $\Gamma$ is equipped with a complete metric and with the corresponding Borel $\sigma$-field, which allows one to employ probability measures on $\Gamma$.

In this work, we follow the statistical approach to stochastic dynamics, see e.g., $[11,12,15]$ and the literature quoted in those articles. In this approach, a model is specified by a Markov 'generator', which acts on observables-appropriate functions $F: \Gamma \rightarrow \mathbb{R}$. For the model considered here, it has the form

$$
(L F)(\gamma)=\sum_{x \in \gamma} \int_{\mathbb{R}^{d}} c(x, y, \gamma)[F(\gamma \backslash x \cup y)-F(\gamma)] d y, \quad \gamma \in \Gamma .
$$

In (1.2) and in the sequel in the corresponding context, we treat each $x \in \mathbb{R}^{d}$ also as a singlepoint configuration $\{x\}$. That is, if $x$ belongs to $\gamma$ (resp. $y$ does not), by $\gamma \backslash x$ (resp. $\gamma \cup y$ ) we mean the configuration which is obtained from $\gamma$ by removing $x$ (resp. by adding $y$ ). The elementary act of the dynamics described by (1.2), which with probability $c(x, y, \gamma) d t$ occurs during the infinitesimal time $d t$, consists in a random change from $\gamma$ to $\gamma \backslash x \cup y$. The rate $c(x, y, \gamma)$ may depend on $z \in \gamma$ with $z \neq x, y$, which is interpreted as an interaction of particles. In this article, we choose

$$
c(x, y, \gamma)=a(x-y) \exp \left(-E^{\phi}(y, \gamma)\right),
$$

where the jump kernel $a: \mathbb{R}^{d} \rightarrow[0,+\infty)=: \mathbb{R}_{+}$is such that $a(x)=a(-x)$ and

$$
\alpha:=\int_{\mathbb{R}^{d}} a(x) d x<\infty .
$$

The second factor in (1.3) describes the interaction, which is supposed to be pair-wise and repulsive. This means that

$$
E^{\phi}(y, \gamma)=\sum_{z \in \gamma} \phi(y-z) \geq 0
$$

where the 'potential' $\phi: \mathbb{R}^{d} \rightarrow \mathbb{R}_{+}$is such that $\phi(x)=\phi(-x)$ and

$$
c_{\phi}:=\int_{\mathbb{R}^{d}}\left(1-e^{-\phi(x)}\right) d x<\infty .
$$

In the sequel, when we speak of the model we consider, we mean the one defined in (1.2)(1.6). We also call it continuum Kawasaki system.

The main reason for us to choose the rates as in (1.3) is that any grand canonical Gibbs measure with potential $\phi$, see e.g., [30], is invariant (even symmetrizing) for the dynamics generated by (1.2) with such rates, see [21]. 
As is usual for Markov dynamics, the 'generator' (1.2) enters the backward Kolmogorov equation

$$
\frac{d}{d t} F_{t}=L F_{t},\left.\quad F_{t}\right|_{t=0}=F_{0},
$$

where, for each $t, F_{t}$ is an observable. In the approach we follow, the states of the system are probability measures on $\Gamma$, and hence $\int_{\Gamma} F d \mu$ can be considered as the value of observable $F$ in state $\mu$. This pairing allows one to define also the corresponding forward Kolmogorov or Fokker-Planck equation

$$
\frac{d}{d t} \mu_{t}=L^{*} \mu_{t},\left.\quad \mu_{t}\right|_{t=0}=\mu_{0}
$$

The evolutions described by (1.2) and (1.8) are mutually dual in the sense that

$$
\int_{\Gamma} F_{t} d \mu_{0}=\int_{\Gamma} F_{0} d \mu_{t} .
$$

Thus, the Cauchy problem in (1.8) determines the evolution of states of our model. If we were able to solve it for all possible probability measures as initial conditions, we could construct a Markov process on $\Gamma$. For nontrivial models, however, including that considered in this work, this is far beyond the possibilities of the available technical tools. The main reason for this is that the configuration space $\Gamma$ has a complex topological structure. Furthermore, the mere existence of the process related to (1.8) would not be enough for drawing conclusions on the collective behavior of the considered system. The basic idea of the approach which we follow is to solve (1.8) not for all possible $\mu_{0}$, but only for those belonging to a properly chosen class of probability measures on $\Gamma$. It turns out that even with such restrictions the direct solving (1.8) is also unattainable, at least so far. Then the solution in question is obtained by employing the so called moment or correlation functions. Similarly as a probability measure on $\mathbb{R}$ is characterized by its moments, a probability measure on $\Gamma$ can be characterized by its correlation functions. Of course, as not every measure on $\mathbb{R}$ has all moments, not every measure on $\Gamma$ possesses correlation functions. The mentioned restriction in the choice of $\mu_{0}$ takes into account, among others, also this issue.

By certain combinatoric calculations, one transforms (1.8) into the following Cauchy problem

$$
\frac{d}{d t} k_{t}=L^{\Delta} k_{t},\left.\quad k_{t}\right|_{t=0}=k_{0},
$$

where $k_{0}$ is the correlation function of $\mu_{0}$. Note that the equation in (1.9) is, in fact, an infinite chain of coupled linear equations. Then the construction of the evolution of states $\mu_{0} \mapsto \mu_{t}$ is performed by: (a) solving (1.9) with $k_{0}=k_{\mu_{0}}$; (b) proving that, for each $t$, there exists a unique probability measure $\mu_{t}$ such that $k_{t}=k_{\mu_{t}}$. This way of constructing the evolution of states is, in a sense, analogous to that suggested by Bogoliubov [2] in the statistical approach to the Hamiltonian dynamics of large systems of interacting physical particles, cf. [4, 16,23] and also a review in [7]. In the theory of such systems, the equation analogous to (1.9) is called $B B G K Y$ chain [7].

The description based on (1.8) or (1.9) is microscopic since one deals with coordinates of individual particles; cf. the Introduction in [29]. More coarse-grained levels are mesoand macroscopic ones. They are attained by appropriate space and time scalings [29,31]. Of course, certain details of the system's behavior are then lost. Kinetic equations provide a space-dependent mean-field-like approximate description of the evolution of infinite particle 
systems. For systems of physical particles, such an equation is the Boltzmann equation related to the BBGKY chain, cf. Sect. 6 in [7] and also [29,31]. Nowadays, a mathematically consistent way of constructing the mesoscopic description based on kinetic equations is the procedure analogous to the Vlasov scaling in plasma physics, see [12]. In its framework, we obtain from (1.9) a new chain of linear equations for limiting 'correlation functions' $r_{t}$, called Vlasov hierarchy. Note that these $r_{t}$ may not be correlation functions at all but they have one important property. Namely, if the initial state $\mu_{0}$ is the Poisson measure with density $\varrho_{0}$, then $r_{t}$ is the correlation function for the Poisson measure $\mu_{t}$ with the density $\varrho_{t}$ which solves the corresponding kinetic equation.

In the present article, we aim at:

- constructing the evolution of states $\mu_{0} \mapsto \mu_{t}$ of the model (1.2), (1.3) by solving (1.9) and then by identifying its solution with a unique $\mu_{t}$;

- deriving rigorously the limiting Vlasov hierarchy, which includes also the convergence of rescaled correlation functions to the limiting functions $r_{t}$, as well as deriving the kinetic equation;

- studying the solvability of the kinetic equation.

Let us make some comments. When speaking of the evolution of states, one might distinguish between equilibrium and non-equilibrium cases. The equilibrium evolution is built with the help of the reversible measures, if such exist for the considered model, and with the corresponding Dirichlet forms. Recall that, for the choice as in (1.3), such reversible measures are grand canonical Gibbs measures. The result is a stationary Markov process, see [21] where a version of the model studied in this work was considered. Note that in this framework, the evolution is restricted to the set of states which are absolutely continuous with respect to the corresponding Gibbs measures. The non-equilibrium evolution, where initial states can be "far away" from equilibrium, is much more interesting and much more complex-for the model considered in this work, it has been constructed for noninteracting particles only, see [22]. In this article, we go further in this direction and construct the non-equilibrium evolution for the continuum Kawasaki system with repulsion. Results similar to those presented here were obtained for a continuum Glauber model in [9], and for a spatial ecological model in [10].

There exists a rich theory of interacting particle systems based on continuous time Markov processes, which studies so called lattice models, see [24] and Part II of [31], and also [25] for the latest results. The essential common feature of these models is that the particles are distributed over a discrete set (lattice), typically $\mathbb{Z}^{d}$. However, in many real-world applications, such as population biology or spatial ecology, the habitat, i.e., the space where the particles are placed, should essentially be continuous, cf. [26], which we take into account in this work. In statistical physics, a lattice model of 'hopping spins' was put forward in [17], see also a review in [18]. There exists an extended theory of interacting particles hopping over $\mathbb{Z}^{d}$, cf. [31, Sect. 1 in Part II], and also, e.g., [3,8] for some aspects of the recent development. However, this theory cannot be applied to continuum Kawasaki systems for a number of reasons. One of which is that a bounded $K \subset \mathbb{R}^{d}$ can contain arbitrary number of particles, whereas the number of particles contained in a bounded $K \subset \mathbb{Z}^{d}$ is at most $|K|$.

\subsection{The Overview of the Results}

The microscopic description is performed in Sect. 3 in two steps. First, we prove that, for a given correlation function $k_{0}$, the problem (1.9) has a unique classical solution $k_{t}$ on a bounded time interval $[0, T)$ and in a Banach space, somewhat bigger than that containing 
$k_{0}$, cf. Theorems 3.1 and 3.2. Here bigger means that the initial space is a proper subspace of the latter. The parameter $T>0$ is related to the 'difference' between the spaces. The main characteristic feature of both Banach spaces is that if their elements are correlation functions of some probability measures on $\Gamma$, then these measures are sub-Poissonian, cf. Definition 2.3 and Remark 2.4. This latter property is important in view of the mesoscopic description which we construct subsequently, cf. Remark 4.1. The restriction of the evolution $k_{0} \mapsto k_{t}$ to a bounded time interval is because we failed to apply to (1.9) semigroup methods, or similar techniques, which would allow for solving this equation on $[0,+\infty)$ in the mentioned Banach spaces. Our method is based on Ovcyannikov's observation, cf. [6, pp. 9-13] and [33], that an unbounded operator can be redefined as a bounded one acting, however, from a 'smaller' to a 'bigger' space, both belonging to a scale of Banach spaces, indexed by $\vartheta \in \mathbb{R}$. The essential fact here is that the norm of such a bounded operator has an upper bound proportional to $\left(\vartheta^{\prime \prime}-\vartheta^{\prime}\right)^{-1}$, see (3.18). This implies that the expansion for $k_{t}$ in powers of $t$ converges for $t \in[0, T)$, cf. (3.19) and (3.21). Second, we prove that the evolution $k_{0} \mapsto k_{t}$ corresponds to the evolution $\mu_{0} \mapsto \mu_{t}$ of uniquely determined probability measures on $\Gamma$ in the following sense. In Theorem 3.8, we show that if $k_{0}$ is the correlation function of a subPoissonian measure $\mu_{0}$, then, for each $t \in(0, T), k_{t}$ is also a correlation function for a unique sub-Poissonian measure $\mu_{t}$. The proof is based on the approximation of states of the infinite system by probability measures on $\Gamma$ supported on the set of finite configurations $\Gamma_{0} \subset \Gamma$ (we call such measures $\Gamma_{0}$-states). The evolution of the latter states can be derived directly from (1.8), which we perform in Theorem 3.7. It is described by a stochastic semigroup constructed with the help of a version of Miyadera's theorem obtained in [32]. Then we prove that the correlation functions of the mentioned states supported on $\Gamma_{0}$ weakly converge to the solution $k_{t}$, which implies that it has the positivity property as in (3.38), which by Proposition 2.2 yields that $k_{t}$ is also a correlation function for a unique state.

The mesoscopic description is performed in Sect. 4 in the framework of the scaling method developed in [12]. First, we derive an analog of (1.9) for the rescaled correlation functions, that is, the Cauchy problem in (4.5). This problem contains the scaling parameter $\varepsilon>0$, which is supposed to tend to zero in the mesoscopic limit. In this limit, we obtain another Cauchy problem, given in (4.12). By the results of Sect. 3, we readily prove the existence of classical solutions of both (4.5) and (4.12). The essence of the scaling technique which we use is that the evolution $r_{0} \mapsto r_{t}$ obtained from (4.12) preserves the set of correlation functions of Poisson measures, cf. Lemma 4.3. Then the density $\varrho_{t}$ that corresponds to $r_{t}$ satisfies the kinetic equation (4.13), which we then transform into an integral equation, cf. (4.15). For its eventual solutions, by the Gronwall inequality we obtain an a priori bound, cf. (4.16), (4.17), by means of which we prove the existence of a unique solution of both (4.13) and (4.15) on $[0,+\infty)$, which implies the global evolution $r_{0} \mapsto r_{t}$, cf. Theorem 4.5. Finally, in Theorem 4.6 we show that the rescaled correlation functions converge to the Poisson correlation functions $r_{t}$ as $\varepsilon \rightarrow 0^{+}$, uniformly on compact subsets of $[0, T)$. This result links both micro- and mesoscopic evolutions constructed in this work.

Let us mention some open problems related to the model studied in this work. The existence of the global mesoscopic evolution does not, however, imply that the restriction of the microscopic evolution to a bounded time interval is only a technical problem. One cannot exclude that, due to an infinite number of jumps, $k_{t}$ finally leaves any space of the type of (3.10). It is still unclear whether the global evolution $k_{0} \mapsto k_{t}$ exists in any of Banach spaces reasonably bigger than those used in Theorems 3.1 and 3.2.

A very interesting problem, in the spirit of the philosophy of Cox [5], is to relate the rate of convergence in (3.54) to the value of $t$, which determines the space in which $k_{t}$ lies, cf. Theorem 3.2. Another open problem is the existence of globally bounded solutions of the 
kinetic equation (4.13). It can be proven that, for a local repulsion, this is the case. Namely, if $\phi$ in (4.13) is such that $(\phi * \varrho)(x)=\varkappa \varrho(x)$ for all $x$ and some $x \geq 0$, and if $\varrho_{0}$ is a bounded continuous function on $\mathbb{R}^{d}$, then the solution $\varrho_{t}$ is also a continuous function, cf. Corollary 3.3, such that $\varrho_{t} \leq \sup _{x \in \mathbb{R}^{d}} \varrho_{0}(x)+\epsilon$ for all $t>0$ and any $\epsilon>0$. From this one can see how important can be the relation between the radii of the jump kernel $a$ and of the repulsion potential $\phi$.

\section{The Basic Notions}

In this paper, we work in the approach of $[10,11,13-15,19]$ where all the relevant details can be found.

\subsection{The Configuration Spaces}

By $\mathcal{B}\left(\mathbb{R}^{d}\right)$ and $\mathcal{B}_{\mathrm{b}}\left(\mathbb{R}^{d}\right)$ we denote the sets of all Borel and all bounded Borel subsets of $\mathbb{R}^{d}$, respectively. The configuration space $\Gamma$ is

$$
\Gamma=\left\{\gamma \subset \mathbb{R}^{d}:|\gamma \cap K|<\infty \text { for any compact } K \subset \mathbb{R}^{d}\right\} .
$$

Each $\gamma \in \Gamma$ can be identified with the following positive Radom measure

$$
\gamma(d x)=\sum_{y \in \gamma} \delta_{y}(d x) \in \mathcal{M}\left(\mathbb{R}^{d}\right),
$$

where $\delta_{y}$ is the Dirac measure centered at $y$, and $\mathcal{M}\left(\mathbb{R}^{d}\right)$ denotes the set of all positive Radon measures on $\mathcal{B}\left(\mathbb{R}^{d}\right)$. This allows one to consider $\Gamma$ as a subset of $\mathcal{M}\left(\mathbb{R}^{d}\right)$, and hence to endow it with the vague topology. The latter is the weakest topology in which all the maps

$$
\Gamma \ni \gamma \mapsto \int_{\mathbb{R}^{d}} f(x) \gamma(d x)=\sum_{x \in \gamma} f(x), \quad f \in C_{0}\left(\mathbb{R}^{d}\right),
$$

are continuous. Here $C_{0}\left(\mathbb{R}^{d}\right)$ stands for the set of all continuous functions $f: \mathbb{R}^{d} \rightarrow \mathbb{R}$ which have compact support. The vague topology on $\Gamma$ admits a metrization, which turns it into a complete and separable (Polish) space, see, e.g., [20, Theorem 3.5]. By $\mathcal{B}(\Gamma)$ we denote the corresponding Borel $\sigma$-field.

For $n \in \mathbb{N}_{0}:=\mathbb{N} \cup\{0\}$, the set of $n$-particle configurations in $\mathbb{R}^{d}$ is

$$
\Gamma^{(0)}=\{\emptyset\}, \quad \Gamma^{(n)}=\left\{\eta \subset \mathbb{R}^{d}:|\eta|=n\right\}, \quad n \in \mathbb{N} .
$$

For $n \geq 2, \Gamma^{(n)}$ can be identified with the symmetrization of the set

$$
\left\{\left(x_{1}, \ldots, x_{n}\right) \in\left(\mathbb{R}^{d}\right)^{n}: x_{i} \neq x_{j}, \text { for } i \neq j\right\} \subset\left(\mathbb{R}^{d}\right)^{n},
$$

which allows one to introduce the corresponding topology and hence the Borel $\sigma$-field $\mathcal{B}\left(\Gamma^{(n)}\right)$. The set of finite configurations is

$$
\Gamma_{0}:=\bigsqcup_{n \in \mathbb{N}_{0}} \Gamma^{(n)} .
$$

We equip it with the topology of the disjoint union and hence with the Borel $\sigma$-field $\mathcal{B}\left(\Gamma_{0}\right)$. Obviously, $\Gamma_{0}$ is a subset of $\Gamma$, cf. (1.1). However, the topology just mentioned and that 
induced from $\Gamma$ do not coincide. At the same time, $\Gamma_{0} \in \mathcal{B}(\Gamma)$. In the sequel, by $\Lambda$ we denote a bounded subset of $\mathbb{R}^{d}$, that is, we always mean $\Lambda \in \mathcal{B}_{\mathrm{b}}\left(\mathbb{R}^{d}\right)$. For such $\Lambda$, we set

$$
\Gamma_{\Lambda}=\{\gamma \in \Gamma: \gamma \subset \Lambda\}
$$

Clearly, $\Gamma_{\Lambda}$ is also a measurable subset of $\Gamma_{0}$ and the following holds

$$
\Gamma_{\Lambda}=\bigsqcup_{n \in \mathbb{N}_{0}}\left(\Gamma^{(n)} \cap \Gamma_{\Lambda}\right),
$$

which allows one to equip $\Gamma_{\Lambda}$ with the topology induced by that of $\Gamma_{0}$. Let $\mathcal{B}\left(\Gamma_{\Lambda}\right)$ be the corresponding Borel $\sigma$-field. It is clear that, for $A \in \mathcal{B}\left(\Gamma_{0}\right), \Gamma_{\Lambda} \cap A \in \mathcal{B}\left(\Gamma_{\Lambda}\right)$. It can be proven, see Lemma 1.1 and Proposition 1.3 in [27], that

$$
\mathcal{B}\left(\Gamma_{\Lambda}\right)=\left\{\Gamma_{\Lambda} \cap A: A \in \mathcal{B}(\Gamma)\right\}
$$

and hence

$$
\mathcal{B}\left(\Gamma_{0}\right)=\left\{A \in \mathcal{B}(\Gamma): A \subset \Gamma_{0}\right\}
$$

Next, we define the projection

$$
\Gamma \ni \gamma \mapsto p_{\Lambda}(\gamma)=\gamma_{\Lambda}:=\gamma \cap \Lambda, \quad \Lambda \in \mathcal{B}_{\mathrm{b}}\left(\mathbb{R}^{d}\right)
$$

It is known [1, p. 451] that $\mathcal{B}(\Gamma)$ is the smallest $\sigma$-algebra of subsets of $\Gamma$ such that the maps $p_{\Lambda}$ with all $\Lambda \in \mathcal{B}_{\mathrm{b}}\left(\mathbb{R}^{d}\right)$ are $\mathcal{B}(\Gamma) / \mathcal{B}\left(\Gamma_{\Lambda}\right)$ measurable. This means that $(\Gamma, \mathcal{B}(\Gamma))$ is the projective limit of the measurable spaces $\left(\Gamma_{\Lambda}, \mathcal{B}\left(\Gamma_{\Lambda}\right)\right), \Lambda \in \mathcal{B}_{\mathrm{b}}\left(\mathbb{R}^{d}\right)$. A set $A \in \mathcal{B}\left(\Gamma_{0}\right)$ is said to be bounded if

$$
A \subset \bigsqcup_{n=0}^{N} \Gamma_{\Lambda}^{(n)}
$$

for some $\Lambda \in \mathcal{B}_{\mathrm{b}}\left(\mathbb{R}^{d}\right)$ and $N \in \mathbb{N}$. The smallest $\Lambda$ such that $A \subset \Gamma_{\Lambda}$ will be called the support of $A$.

\subsection{Measures and Functions}

Given $n \in \mathbb{N}$, by $m^{(n)}$ we denote the restriction of the Lebesgue product measure $d x_{1} d x_{2} \cdots d x_{n}$ to $\left(\Gamma^{(n)}, \mathcal{B}\left(\Gamma^{(n)}\right)\right)$. The Lebesgue-Poisson measure with intensity $\varkappa>0$ is a measure on $\left(\Gamma_{0}, \mathcal{B}\left(\Gamma_{0}\right)\right)$ defined by

$$
\lambda_{\varkappa}=\delta_{\emptyset}+\sum_{n=1}^{\infty} \frac{\varkappa^{n}}{n !} m^{(n)} .
$$

For $\Lambda \in \mathcal{B}_{\mathrm{b}}\left(\mathbb{R}^{d}\right)$, the restriction of $\lambda_{\varkappa}$ to $\Gamma_{\Lambda}$ will be denoted by $\lambda_{\varkappa}^{\Lambda}$. This is a finite measure on $\mathcal{B}\left(\Gamma_{\Lambda}\right)$ such that

$$
\lambda_{\varkappa}^{\Lambda}\left(\Gamma_{\Lambda}\right)=\exp [\varkappa m(\Lambda)]
$$

where $m(\Lambda):=m^{(1)}(\Lambda)$ is the Lebesgue measure of $\Lambda$. Then

$$
\pi_{\varkappa}^{\Lambda}:=\exp (-\varkappa m(\Lambda)) \lambda_{\varkappa}^{\Lambda}
$$


is a probability measure on $\mathcal{B}\left(\Gamma_{\Lambda}\right)$. It can be shown [1] that the family $\left\{\pi_{\varkappa}^{\Lambda}\right\}_{\Lambda \in \mathcal{B}_{\mathrm{b}}\left(\mathbb{R}^{d}\right)}$ is consistent, and hence there exists a unique probability measure, $\pi_{\varkappa}$, on $\mathcal{B}(\Gamma)$ such that

$$
\pi_{\varkappa}^{\Lambda}=\pi_{\varkappa} \circ p_{\Lambda}^{-1}, \quad \Lambda \in \mathcal{B}_{\mathrm{b}}\left(\mathbb{R}^{d}\right),
$$

where $p_{\Lambda}$ is the same as in (2.5). This $\pi_{\varkappa}$ is called the Poisson measure. The Poisson measure $\pi_{\varrho}$ corresponding to the density $\varrho: \mathbb{R} \rightarrow \mathbb{R}_{+}$is introduced by means of the measure $\lambda_{\varrho}$, defined as in (2.7) with $\varkappa m$ replaced by $m_{\varrho}$, where, for $\Lambda \in \mathcal{B}_{\mathrm{b}}\left(\mathbb{R}^{d}\right)$,

$$
m_{\varrho}(\Lambda):=\int_{\Lambda} \varrho(x) d x
$$

which is supposed to be finite. Then $\pi_{\varrho}$ is defined by its projections

$$
\pi_{\varrho}^{\Lambda}=\exp \left(-m_{\varrho}(\Lambda)\right) \lambda_{\varrho}^{\Lambda} .
$$

For $\varkappa=1$, we shall drop the subscript and consider the Lebesgue-Poisson measure $\lambda$ and the Poisson measure $\pi$.

For a measurable $f: \mathbb{R}^{d} \rightarrow \mathbb{R}$ and $\eta \in \Gamma_{0}$, the Lebesgue-Poisson exponent is

$$
e(f, \eta)=\prod_{x \in \eta} f(x), \quad e(f, \emptyset)=1 .
$$

Clearly, $e(f, \cdot) \in L^{1}\left(\Gamma_{0}, d \lambda\right)$ for any $f \in L^{1}\left(\mathbb{R}^{d}\right):=L^{1}\left(\mathbb{R}^{d}, d x\right)$, and

$$
\int_{\Gamma_{0}} e(f, \eta) \lambda(d \eta)=\exp \left\{\int_{\mathbb{R}^{d}} f(x) d x\right\} .
$$

By $B_{\mathrm{bs}}\left(\Gamma_{0}\right)$ we denote the set of all bounded measurable functions $G: \Gamma_{0} \rightarrow \mathbb{R}$, which have bounded supports. That is, each such $G$ is the zero function on $\Gamma_{0} \backslash A$ for some bounded $A$, cf. (2.6). Note that any measurable $G: \Gamma_{0} \rightarrow \mathbb{R}$ is in fact a sequence of measurable symmetric functions $G^{(n)}:\left(\mathbb{R}^{d}\right)^{n} \rightarrow \mathbb{R}$ such that, for $\eta=\left\{x_{1}, \ldots, x_{n}\right\}, G(\eta)=G^{(n)}\left(x_{1}, \ldots, x_{n}\right)$. We say that $F: \Gamma \rightarrow \mathbb{R}$ is a cylinder function if there exists $\Lambda \in \mathcal{B}_{\mathrm{b}}\left(\mathbb{R}^{d}\right)$ and $G: \Gamma_{\Lambda} \rightarrow \mathbb{R}$ such that $F(\gamma)=G\left(\gamma_{\Lambda}\right)$ for all $\gamma \in \Gamma$. By $\mathcal{F}_{\text {cyl }}(\Gamma)$ we denote the set of all measurable cylinder functions. For $\gamma \in \Gamma$, by writing $\eta \Subset \gamma$ we mean that $\eta \subset \gamma$ and $\eta$ is finite, i.e., $\eta \in \Gamma_{0}$. For $G \in B_{\mathrm{bs}}\left(\Gamma_{0}\right)$, we set

$$
(K G)(\gamma)=\sum_{\eta \Subset \gamma} G(\eta), \quad \gamma \in \Gamma .
$$

Clearly $K$ maps $B_{\text {bs }}\left(\Gamma_{0}\right)$ into $\mathcal{F}_{\text {cyl }}(\Gamma)$ and is linear and positivity preserving. This map plays an important role in the theory of configuration spaces, cf. [19].

By $\mathcal{M}^{1}(\Gamma)$ we denote the set of all probability measures on $(\Gamma, \mathcal{B}(\Gamma))$, and let $\mathcal{M}_{\mathrm{fm}}^{1}(\Gamma)$ denote the subset of $\mathcal{M}^{1}(\Gamma)$ consisting of all measures which have finite local moments, that is, for which

$$
\int_{\Gamma}\left|\gamma_{\Lambda}\right|^{n} \mu(d \gamma)<\infty \text { for all } n \in \mathbb{N} \text { and } \Lambda \in \mathcal{B}_{\mathrm{b}}\left(\mathbb{R}^{d}\right) .
$$

Definition 2.1 A measure $\mu \in \mathcal{M}_{\mathrm{fm}}^{1}(\Gamma)$ is said to be locally absolutely continuous with respect to the Poisson measure $\pi$ if, for every $\Lambda \in \mathcal{B}_{\mathrm{b}}\left(\mathbb{R}^{d}\right)$, the projection 


$$
\mu^{\Lambda}:=\mu \circ p_{\Lambda}^{-1}
$$

is absolutely continuous with respect to $\pi^{\Lambda}$ and hence with respect to $\lambda^{\Lambda}$, see (2.8).

A measure $\chi$ on $\left(\Gamma_{0}, \mathcal{B}\left(\Gamma_{0}\right)\right)$ is said to be locally finite if $\chi(A)<\infty$ for every bounded measurable $A \subset \Gamma_{0}$. By $\mathcal{M}_{\mathrm{lf}}\left(\Gamma_{0}\right)$ we denote the set of all such measures. Let a measurable $A \subset \Gamma_{0}$ be bounded, and let $\mathbb{I}_{A}$ be its indicator function on $\Gamma_{0}$. Then $\mathbb{I}_{A}$ is in $B_{\mathrm{bs}}\left(\Gamma_{0}\right)$, and hence one can apply (2.13). For $\mu \in \mathcal{M}_{\mathrm{fm}}^{1}(\Gamma)$, we let

$$
\chi_{\mu}(A)=\int_{\Gamma}\left(K \mathbb{I}_{A}\right)(\gamma) \mu(d \gamma),
$$

which uniquely determines a measure $\left.\chi_{\mu} \in \mathcal{M}_{1 \mathrm{f}}\left(\Gamma_{0}\right)\right)$. It is called the correlation measure for $\mu$. For instance, let $A \subset \Gamma^{(n)} \subset \Gamma_{0}$ and let $\Lambda \in \mathcal{B}_{\mathrm{b}}\left(\mathbb{R}^{d}\right)$ be the support of $A$, cf. (2.6). Then $\left(K \mathbb{I}_{A}\right)(\gamma)$ is the number of distinct $n$-particle sub-configurations of $\gamma$ contained in $\Lambda$, and thus $\chi_{\mu}(A)$ is the expected number of such sub-configurations in $\Lambda$ in state $\mu$. In particular, if $A \subset \Gamma^{(1)}$, then $\chi_{\mu}(A)$ is just the expected number of particles in state $\mu$ contained in $\Lambda$.

The equation (2.15) defines a map $\left.K^{*}: \mathcal{M}_{\mathrm{fm}}^{1}(\Gamma) \rightarrow \mathcal{M}_{\mathrm{lf}}\left(\Gamma_{0}\right)\right)$ such that $K^{*} \mu=\chi_{\mu}$. In particular, $K^{*} \pi=\lambda$. It is known, see [19, Proposition 4.14], that $\chi_{\mu}$ is absolutely continuous with respect to $\lambda$ if $\mu$ is locally absolutely continuous with respect to $\pi$. In this case, we have that, for any $\Lambda \in \mathcal{B}_{\mathrm{b}}\left(\mathbb{R}^{d}\right)$ and $\lambda^{\Lambda}$-almost all $\eta \in \Gamma_{\Lambda}$,

$$
\begin{aligned}
k_{\mu}(\eta)=\frac{d \chi_{\mu}}{d \lambda}(\eta) & =\int_{\Gamma_{\Lambda}} \frac{d \mu^{\Lambda}}{d \pi^{\Lambda}}(\eta \cup \gamma) \pi^{\Lambda}(d \gamma) \\
& =\int_{\Gamma_{\Lambda}} \frac{d \mu^{\Lambda}}{d \lambda^{\Lambda}}(\eta \cup \xi) \lambda^{\Lambda}(d \xi) .
\end{aligned}
$$

The Radon-Nikodym derivative $k_{\mu}$ is called the correlation function corresponding to the measure $\mu$. As all real-valued measurable functions on $\Gamma_{0}$, each $k_{\mu}$ is the collection of measurable $k_{\mu}^{(n)}:\left(\mathbb{R}^{d}\right)^{n} \rightarrow \mathbb{R}$ such that $k_{\mu}^{(0)} \equiv 1$, and $k_{\mu}^{(n)}, n \geq 2$, are symmetric. In particular, $k_{\mu}^{(1)}$ is the particle's density in state $\mu$, cf. (2.15).

Recall that by $B_{\mathrm{bs}}\left(\Gamma_{0}\right)$ we denote the set of all bounded measurable functions $G: \Gamma_{0} \rightarrow \mathbb{R}$, which have bounded supports. We also set

$$
B_{\mathrm{bs}}^{+}\left(\Gamma_{0}\right)=\left\{G \in B_{\mathrm{bs}}\left(\Gamma_{0}\right):(K G)(\gamma) \geq 0\right\} .
$$

The following fact is known, see Theorems 6.1, 6.2 and Remark 6.3 in [19].

Proposition 2.2 Suppose $\left.\chi \in \mathcal{M}_{\mathrm{lf}}\left(\Gamma_{0}\right)\right)$ has the properties

$$
\chi(\{\emptyset\})=1, \quad \int_{\Gamma_{0}} G(\eta) \chi(d \eta) \geq 0,
$$

for each $G \in B_{\mathrm{bs}}^{+}\left(\Gamma_{0}\right)$. Then there exist $\mu \in \mathcal{M}_{\mathrm{fm}}^{1}(\Gamma)$ such that $K^{*} \mu=\chi$. For the uniqueness of such $\mu$, it is enough that the Radon-Nikodym derivative (2.16) of $\chi$ obeys

$$
k(\eta) \leq \prod_{x \in \eta} C_{R}(x)
$$

for all $\eta \in \Gamma_{0}$ and for some locally integrable $C_{R}: \mathbb{R}^{d} \rightarrow \mathbb{R}_{+}$. 
Let $\pi_{\varrho}$ be the Poisson measure as in (2.10), and let $\Lambda$ be the support of a given bounded $A \subset \Gamma_{0}$. If $A \subset \Gamma^{(n)}$, cf. (2.1), then

$$
\chi_{\pi_{\varrho}}(A)=\int_{\Lambda^{n}} \varrho\left(x_{1}\right) \cdots \varrho\left(x_{n}\right) d x_{1} \cdots d x_{n}=\left(\int_{\Lambda} \varrho(x) d x\right)^{n},
$$

which, in particular, means that particles appear in $\Lambda$ independently. In this case,

$$
k_{\pi_{\varrho}}(\eta)=e(\varrho, \eta)
$$

where $e$ is as in (2.11). In particular,

$$
k_{\pi_{\varrho}}^{(n)}\left(x_{1}, \ldots, x_{n}\right)=\varrho\left(x_{1}\right) \cdots \varrho\left(x_{n}\right), \quad n \in \mathbb{N} .
$$

Definition 2.3 A locally absolutely continuous measure $\mu \in \mathcal{M}_{\mathrm{fm}}^{1}(\Gamma)$, cf. Definition 2.1, is called sub-Poissonian if its correlation function $k_{\mu}$ obeys (2.19) for some locally integrable $C_{R}: \mathbb{R}^{d} \rightarrow \mathbb{R}_{+}$.

Remark 2.4 If $\mu$ is sub-Poissonian and $A$ is as in (2.20), then

$$
\chi_{\mu}(A) \leq C^{n}\left(\int_{\Lambda} k_{\mu}^{(1)}(x) d x\right)^{n}
$$

for some $C>0$. That is, the correlation measure is controlled by the density in this case. For instance, if one knows that $k_{\mu_{t}}^{(1)}$ does not explode for all $t>0$, then so does $k_{\mu_{t}}$, and hence $\mu_{t}$ exists for all $t>0$. A faster increase of $\chi_{\mu}(A)$, e.g., as $n$ !, can be interpreted as clustering in state $\mu$.

Finally, we present the following integration rule, cf. [11, Lemma 2.1],

$$
\int_{\Gamma_{0}} \sum_{\xi \subset \eta} H(\xi, \eta \backslash \xi, \eta) \lambda(d \eta)=\int_{\Gamma_{0}} \int_{\Gamma_{0}} H(\xi, \eta, \eta \cup \xi) \lambda(d \xi) \lambda(d \eta),
$$

which holds for any appropriate function $H$.

\section{Microscopic Dynamics}

In view of the fact that $\Gamma$ contains also infinite configurations, the direct construction of the evolution based on (1.7) and (1.2) cannot be done, and thus we pass to the description based on correlation functions, cf. (1.9) and (2.16). The 'generator' in (1.9) has the form, cf. [15, Eq. (4.8)]

$$
\begin{aligned}
\left(L^{\Delta} k\right)(\eta)=\sum_{y \in \eta} \int_{\mathbb{R}^{d}} a(x-y) e\left(\tau_{y}, \eta \backslash y \cup x\right) \\
\\
\quad \times\left(\int_{\Gamma_{0}} e\left(t_{y}, \xi\right) k(\xi \cup x \cup \eta \backslash y) \lambda(d \xi)\right) d x
\end{aligned}
$$




$$
\begin{aligned}
& -\int_{\Gamma_{0}} k(\xi \cup \eta)\left(\sum_{x \in \eta} \int_{\mathbb{R}^{d}} a(x-y) e\left(\tau_{y}, \eta\right)\right. \\
& \left.\quad \times e\left(t_{y}, \xi\right) d y\right) \lambda(d \xi) .
\end{aligned}
$$

Here $e$ is as in (2.11) and

$$
t_{x}(y)=e^{-\phi(x-y)}-1, \quad \tau_{x}(y)=t_{x}(y)+1 .
$$

We shall also consider the following auxiliary evolution $G_{0} \mapsto G_{t}$, dual to that $k_{0} \mapsto k_{t}$ described by (1.9) and (3.1). The duality is understood in the sense

$$
\left\langle\left\langle G_{t}, k_{0}\right\rangle\right\rangle=\left\langle\left\langle G_{0}, k_{t}\right\rangle\right\rangle,
$$

where

$$
\langle\langle G, k\rangle\rangle:=\int_{\Gamma_{0}} G(\eta) k(\eta) \lambda(d \eta),
$$

and $\lambda$ is the Lebesgue-Poisson measure defined in (2.7) with $\varkappa=1$. The 'generators' are related to each other by

$$
\left\langle\left\langle G, L^{\Delta} k\right\rangle\right\rangle=\langle\langle\widehat{L} G, k\rangle\rangle .
$$

Then the equation dual to (1.9) is

$$
\frac{d}{d t} G_{t}=\widehat{L} G_{t},\left.\quad G_{t}\right|_{t=0}=G_{0}
$$

with, cf. [15, Eq. (4.7)],

$$
\begin{aligned}
(\widehat{L} G)(\eta)= & \sum_{\xi \subset \eta} \sum_{x \in \xi} \int_{\mathbb{R}^{d}} a(x-y) e\left(\tau_{y}, \xi\right) \\
& \quad \times e\left(t_{y}, \eta \backslash \xi\right)[G(\xi \backslash x \cup y)-G(\xi)] d y .
\end{aligned}
$$

\subsection{The Evolution of Correlation Functions}

We consider (1.9) with $L^{\Delta}$ given in (3.1). To place this problem in the right context we introduce the following Banach spaces. Recall that a function $G: \Gamma_{0} \rightarrow \mathbb{R}$ is a sequence of $G^{(n)}:\left(\mathbb{R}^{d}\right)^{n} \rightarrow \mathbb{R}, n \in \mathbb{N}_{0}$, where $G^{(0)}$ is constant and all $G^{(n)}, n \geq 2$, are symmetric. Let $k: \Gamma_{0} \rightarrow \mathbb{R}$ be such that $k^{(n)} \in L^{\infty}\left(\left(\mathbb{R}^{d}\right)^{n}\right)$, for $n \in \mathbb{N}$. For this $k$ and $\vartheta \in \mathbb{R}$, we set

$$
\|k\|_{\vartheta}=\sup _{n \in \mathbb{N}_{0}} v_{n}(k) \exp (\vartheta n)
$$

where

$$
v_{0}(k)=\left|k^{(0)}\right|, \quad v_{n}(k)=\left\|k^{(n)}\right\|_{L^{\infty}\left(\left(\mathbb{R}^{d}\right)^{n}\right)}, \quad n \in \mathbb{N} .
$$

Then

$$
\mathcal{K}_{\vartheta}:=\left\{k: \Gamma_{0} \rightarrow \mathbb{R}:\|k\|_{\vartheta}<\infty\right\},
$$

is a real Banach space with norm (3.8) and usual point-wise linear operations. Note that $\left\{\mathcal{K}_{\vartheta}: \vartheta \in \mathbb{R}\right\}$ is a scale of Banach spaces in the sense that 


$$
\mathcal{K}_{\vartheta} \subset \mathcal{K}_{\vartheta^{\prime}}, \text { for } \vartheta>\vartheta^{\prime} .
$$

As usual, by a classical solution of (1.9) in $\mathcal{K}_{\vartheta}$ on time interval $I$, we understand a map $I \ni t \mapsto k_{t} \in \mathcal{K}_{\vartheta}$, which is continuous on $I$, continuously differentiable on the interior of $I$, lies in the domain of $L^{\Delta}$, and solves (1.9). Recall that we suppose (1.6) and (1.4).

Theorem 3.1 Given $\vartheta \in \mathbb{R}$ and $T>0$, we let

$$
\vartheta_{0}=\vartheta+2 \alpha T \exp \left(c_{\phi} e^{-\vartheta}\right) .
$$

Then the problem (1.9) with $k_{0} \in \mathcal{K}_{\vartheta_{0}}$ has a unique classical solution $k_{t} \in \mathcal{K}_{\vartheta}$ on $[0, T)$.

According to the above theorem, for arbitrary $T>0$ and $\vartheta$, one can pick the initial space such that the evolution $k_{0} \mapsto k_{t}$ lasts in $\mathcal{K}_{\vartheta}$ until $t<T$. On the other hand, if the initial space is given, the evolution is restricted in time to the interval $[0, T(\vartheta))$ with

$$
T(\vartheta)=\frac{\vartheta_{0}-\vartheta}{2 \alpha} \exp \left(-c_{\phi} e^{-\vartheta}\right) .
$$

Clearly, $T\left(\vartheta_{0}\right)=0$ and $T(\vartheta) \rightarrow 0$ as $\vartheta \rightarrow-\infty$. Hence, there exists $T_{*}=T_{*}\left(\vartheta_{0}, \alpha, c_{\phi}\right)$ such that $T(\vartheta) \leq T_{*}$ for all $\vartheta \in\left(-\infty, \vartheta_{0}\right]$. Set

$$
\vartheta(t)=\sup \left\{\vartheta \in\left(-\infty, \vartheta_{0}\right]: t<T(\vartheta)\right\} .
$$

Then the alternative version of the above theorem can be formulated as follows.

Theorem 3.2 For every $\vartheta_{0} \in \mathbb{R}$, there exists $T_{*}=T_{*}\left(\vartheta_{0}, \alpha, c_{\phi}\right)$ such that the problem (1.9) with $k_{0} \in \mathcal{K}_{\vartheta_{0}}$ has a unique classical solution $k_{t} \in \mathcal{K}_{\vartheta(t)}$ on $\left[0, T_{*}\right)$.

Proof of Theorem 1 Let $\vartheta \in \mathbb{R}$ be fixed. Set

$$
\operatorname{Dom}\left(L^{\Delta}\right)=\left\{k \in \mathcal{K}_{\vartheta}: L^{\Delta} k \in \mathcal{K}_{\vartheta}\right\}
$$

Given $k$, let $L_{1}^{\Delta} k$ and $L_{2}^{\Delta} k$ denote the first and the second summands in (3.1), respectively. Then, for $\vartheta \leq \vartheta^{\prime}<\vartheta^{\prime \prime}$ and $k \in \mathcal{K}_{\vartheta^{\prime \prime}}$, we have

$$
\begin{aligned}
\left|\left(L_{1}^{\Delta} k\right)(\eta)\right| e^{\vartheta^{\prime}}|\eta| & \\
\leq & \sum_{y \in \eta} \int_{\mathbb{R}^{d}} d x a(x-y) \int_{\Gamma_{0}} \lambda(d \xi)|k(\eta \backslash y \cup x \cup \xi)| \exp \left(\vartheta^{\prime}|\eta \cup \xi|\right) \\
& \times \exp \left(-\vartheta^{\prime \prime}|\eta \cup \xi|\right) e\left(\left|t_{y}\right|, \xi\right) \mid e^{\vartheta^{\prime}|\eta|} \\
\leq & \sum_{y \in \eta} \int_{\mathbb{R}^{d}} d x a(x-y) \int_{\Gamma_{0}} \lambda(d \xi)\|k\|_{\vartheta^{\prime \prime}} e^{-\vartheta^{\prime \prime}|\xi|} \\
& \times e\left(\left|t_{y}\right|, \xi\right) \mid e^{-|\eta|\left(\vartheta^{\prime \prime}-\vartheta^{\prime}\right)} \\
= & \|k\|_{\vartheta^{\prime \prime}} \alpha \exp \left(e^{-\vartheta^{\prime \prime}} c_{\phi}\right)|\eta| e^{-|\eta|\left(\vartheta^{\prime \prime}-\vartheta^{\prime \prime}\right)} \\
\leq & \|k\|_{\vartheta^{\prime \prime}} \alpha \exp \left(e^{-\vartheta^{\prime \prime}} c_{\phi}\right) \frac{1}{e\left(\vartheta^{\prime \prime}-\vartheta^{\prime}\right)}
\end{aligned}
$$

which holds for $\lambda$-almost all $\eta \in \Gamma_{0}$. In the last line we used (2.12) and the following inequality

$$
\tau e^{-\delta \tau} \leq 1 / e \delta, \text { for all } \tau \geq 0, \quad \delta>0 .
$$


Similarly one estimates also $L_{2}^{\Delta} k$, which finally yields

$$
\left\|L^{\Delta} k\right\|_{\vartheta^{\prime}} \leq \frac{2 \alpha}{e\left(\vartheta^{\prime \prime}-\vartheta^{\prime}\right)} \exp \left(c_{\phi} e^{-\vartheta^{\prime \prime}}\right)\|k\|_{\vartheta^{\prime \prime}}, \quad \vartheta^{\prime \prime}>\vartheta^{\prime}
$$

and hence, cf. (3.11) and (3.15),

$$
\operatorname{Dom}\left(L^{\Delta}\right) \supset \mathcal{K}_{\vartheta^{\prime}}, \quad \text { for all } \vartheta^{\prime}>\vartheta
$$

By (3.16), $L^{\Delta}$ can be defined as a bounded linear operator $L^{\Delta}: \mathcal{K}_{\vartheta^{\prime \prime}} \rightarrow \mathcal{K}_{\vartheta^{\prime}}, \vartheta^{\prime}<\vartheta^{\prime \prime}$, with norm

$$
\left\|L^{\Delta}\right\|_{\vartheta^{\prime \prime} \vartheta^{\prime}} \leq \frac{2 \alpha}{e\left(\vartheta^{\prime \prime}-\vartheta^{\prime}\right)} \exp \left(c_{\phi} e^{-\vartheta^{\prime \prime}}\right) .
$$

Given $k_{0} \in \mathcal{K}_{\vartheta_{0}}$, we seek the solution of (1.9) as the limit of the sequence $\left\{k_{t, n}\right\}_{n \in \mathbb{N}_{0}} \subset \mathcal{K}_{\vartheta}$, where $k_{t, 0}=k_{0}$ and

$$
k_{t, n}=k_{0}+\int_{0}^{t} L^{\Delta} k_{s, n-1} d s, \quad n \in \mathbb{N} .
$$

The latter can be iterated to yield

$$
k_{t, n}=k_{0}+\sum_{m=1}^{n} \frac{1}{m !} t^{m}\left(L^{\Delta}\right)^{m} k_{0} .
$$

Then, for $n, p \in \mathbb{N}$, we have

$$
\left\|k_{t, n}-k_{t, n+p}\right\|_{\vartheta} \leq \sum_{m=n+1}^{n+p} \frac{t^{m}}{m !}\left\|\left(L^{\Delta}\right)^{m}\right\|_{\vartheta_{0} \vartheta}\left\|k_{0}\right\|_{\vartheta_{0}} .
$$

For a given $m \in \mathbb{N}$ and $l=0, \ldots, m$, set $\vartheta_{l}=\vartheta+(m-l) \epsilon, \epsilon=\left(\vartheta_{0}-\vartheta\right) / m$. Then by (3.18) and (3.13), we get

$$
\begin{aligned}
\left\|\left(L^{\Delta}\right)^{m}\right\|_{\vartheta_{0} \vartheta} & \leq \prod_{l=0}^{m}\left\|L^{\Delta}\right\|_{\vartheta_{l} \vartheta_{l+1}} \leq\left(\frac{2 \alpha m \exp \left[c_{\phi} e^{-\vartheta}\right]}{e\left(\vartheta_{0}-\vartheta\right)}\right)^{m} \\
& =\left(\frac{m}{e}\right)^{m} \frac{1}{[T(\vartheta)]^{m}} .
\end{aligned}
$$

Applying the latter estimate in (3.20) we obtain that the sequence $\left\{k_{t}^{(n)}\right\}_{n \in \mathbb{N}}$ converges in $\mathcal{K}_{\vartheta}$ for $|t|<T$, and hence is differentiable, even real analytic, in $\mathcal{K}_{\vartheta}$ on the latter set. From the proof above we see that $\left\{k_{t}^{(n)}\right\}_{n \in \mathbb{N}}$ converges also in $\mathcal{K}_{\vartheta(t)}$ with $\vartheta(t)$ as in (3.14), which proves Theorem 3.2. The latter by (3.17) yields that $k_{t} \in \operatorname{Dom}\left(L^{\Delta}\right)$ for all $t<T(\vartheta)$, which completes the proof of the existence. The uniqueness readily follows by the analyticity just mentioned.

Let now $k_{t}$, as a function of $\eta \in \Gamma_{0}$, be continuous. Then instead of (3.10) we consider

$$
\widetilde{\mathcal{K}}_{\vartheta}=\left\{k \in C\left(\Gamma_{0} \rightarrow \mathbb{R}\right):\|k\|_{\vartheta}<\infty\right\},
$$

endowed with the same norm as in (3.8), (3.9).

Corollary 3.3 Let $\vartheta, T$, and $\vartheta_{0}$ be as in Theorem 3.1. Suppose in addition that the function $\phi$ is continuous. Then the problem (1.9) with $k_{0} \in \widetilde{\mathcal{K}}_{\vartheta_{0}}$ has a unique classical solution $k_{t} \in \widetilde{\mathcal{K}}_{\vartheta}$ on $[0, T)$. 
Now we consider (3.6) in the Banach space

$$
\mathcal{G}_{\vartheta}=L^{1}\left(\Gamma_{0}, e^{-\vartheta|\cdot|} d \lambda\right), \quad \vartheta \in \mathbb{R}
$$

that is, $G \in \mathcal{G}_{\vartheta}$ if $\|G\|_{\vartheta}<\infty$, where

$$
\|G\|_{\vartheta}:=\int_{\Gamma_{0}} \exp (-\vartheta|\eta|)|G(\eta)| \lambda(d \eta) .
$$

Theorem 3.4 Let $\vartheta_{0} \vartheta, T>0$ be as in (3.12). Then the Cauchy problem (3.6) with $G_{0} \in \mathcal{G}_{\vartheta}$ has a unique classical solution $G_{t} \in \mathcal{G}_{\vartheta_{0}}$ on $[0, T)$.

Proof As above, we obtain the solution of (3.6) as the limit of the sequence $\left\{G_{t, n}\right\}_{n \in \mathbb{N}_{0}} \subset \mathcal{G}_{\vartheta_{0}}$, where $G_{t}^{(0)}=G_{0}$ and

$$
G_{t, n}=G_{0}+\sum_{m=1}^{n} \frac{1}{m !} t^{m} \widehat{L}^{m} G_{0} .
$$

For the norm (3.22), from (3.7) similarly as above by (2.23) we get

$$
\|\widehat{L} G\|_{\vartheta^{\prime \prime}} \leq \frac{2 \alpha}{e\left(\vartheta^{\prime \prime}-\vartheta^{\prime}\right)} \exp \left(c_{\phi} e^{-\vartheta^{\prime \prime}}\right)\|G\|_{\vartheta^{\prime}}
$$

This means that $\widehat{L}$ can be defined as a bounded linear operator $\widehat{L}: \mathcal{G}_{\vartheta^{\prime}} \rightarrow \mathcal{G}_{\vartheta^{\prime \prime}}$ with norm

$$
\|\widehat{L}\|_{\vartheta^{\prime} \vartheta^{\prime \prime}} \leq \frac{2 \alpha}{e\left(\vartheta^{\prime \prime}-\vartheta^{\prime}\right)} \exp \left(c_{\phi} e^{-\vartheta}\right)
$$

Then we apply the latter estimate in (3.23) and obtain, for any $p, n \in \mathbb{N}$,

$$
\left\|G_{t, n}-G_{t, n+p}\right\|_{\vartheta} \leq \sum_{m=n+1}^{n+p} \frac{(m / e)^{m}}{m !}\left(\frac{t}{T}\right)^{m} .
$$

The latter estimate yields the proof, as in the case of Theorem 3.1.

Corollary 3.5 Let $k_{0}, k_{t}$, and $G_{0}, G_{t}$ be as in Theorems 3.1 and 3.4, respectively. Then, $c f$. (3.3), the following holds

$$
\left\langle\left\langle G_{0}, k_{t}\right\rangle\right\rangle=\left\langle\left\langle G_{t}, k_{0}\right\rangle\right\rangle
$$

That is, the evolutions described by these Theorems are dual.

Proof By (3.5) and by (3.19) and (3.23), we see that, for all $n \in \mathbb{N}$,

$$
\left\langle\left\langle G_{0}, k_{t, n}\right\rangle\right\rangle=\left\langle\left\langle G_{t, n}, k_{0}\right\rangle\right\rangle .
$$

Then (3.24) is obtained from the latter by passing to the limit $n \rightarrow+\infty$, since we have the norm-convergences of the sequences $\left\{k_{t, n}\right\}$ and $\left\{G_{t, n}\right\}$. 


\subsection{The Evolution of $\Gamma_{0}$-States}

We recall that the set of finite configurations $\Gamma_{0}$, cf. (2.2), is a measurable subset of $\Gamma$. By a $\Gamma_{0}$-state we mean a state $\mu \in \mathcal{M}^{1}(\Gamma)$ such that $\mu\left(\Gamma_{0}\right)=1$. That is, in a $\Gamma_{0}$-state the system consists of a finite number of particles, but this number is random. Each $\Gamma_{0}$-state can be redefined as a probability measure on $\left(\Gamma_{0}, \mathcal{B}\left(\Gamma_{0}\right)\right)$, cf. (2.3) and (2.4). The action of the 'generator' in (1.8) on $\Gamma_{0}$-states can be written down explicitly. Namely, for such a state $\mu$ and $A \in \mathcal{B}\left(\Gamma_{0}\right)$,

$$
\left(L^{*} \mu\right)(A)=-\int_{\Gamma_{0}} \Omega\left(\Gamma_{0}, \eta\right) \mathbb{I}_{A}(\eta) \mu(d \eta)+\int_{\Gamma_{0}} \Omega(A, \eta) \mu(d \eta),
$$

where, cf. (1.3) and (1.5),

$$
\Omega(A, \eta)=\sum_{x \in \eta} \int_{\mathbb{R}^{d}} a(x-y) \exp \left(-E^{\phi}(y, \eta)\right) \mathbb{I}_{A}(\eta \backslash x \cup y) d y,
$$

which is a measure kernel on $\left(\Gamma_{0}, \mathcal{B}\left(\Gamma_{0}\right)\right)$. That is, $\Omega(\cdot, \eta)$ is a measure for all $\eta \in \Gamma_{0}$, and $\Omega(A, \cdot)$ is $\mathcal{B}\left(\Gamma_{0}\right)$-measurable for all $A \in \mathcal{B}\left(\Gamma_{0}\right)$. Note that

$$
\Omega\left(\Gamma_{0}, \eta\right)=\sum_{x \in \eta} \int_{\mathbb{R}^{d}} a(x-y) \exp \left(-E^{\phi}(y, \eta)\right) d y \leq \alpha|\eta|,
$$

which is obtained by (1.4) and the positivity of $\phi$.

Let $\mathcal{M}\left(\Gamma_{0}\right)$ be the Banach space of all signed measures on $\left(\Gamma_{0}, \mathcal{B}\left(\Gamma_{0}\right)\right)$ which have bounded variation. For each $\mu \in \mathcal{M}\left(\Gamma_{0}\right)$, there exist $\beta_{ \pm} \geq 0$ and probability measures $\mu_{ \pm}$such that

$$
\mu=\beta_{+} \mu_{+}-\beta_{-} \mu_{-}, \quad \text { and }\|\mu\|=\beta_{+}+\beta_{-} .
$$

Let $\mathcal{M}_{+}\left(\Gamma_{0}\right)$ be the cone of positive elements of $\mathcal{M}\left(\Gamma_{0}\right)$, for which $\|\mu\|=\mu\left(\Gamma_{0}\right)$. Then we define, cf. (3.27),

$$
\operatorname{Dom}\left(L^{*}\right)=\left\{\mu \in \mathcal{M}\left(\Gamma_{0}\right): \Omega\left(\Gamma_{0}, \cdot\right) \mu \in \mathcal{M}\left(\Gamma_{0}\right)\right\} .
$$

Recall that a $C_{0}$-semigroup $\left\{S_{\mu}(t)\right\}_{t \geq 0}$ of bounded operators in $\mathcal{M}\left(\Gamma_{0}\right)$ is called stochastic if each $S_{\mu}(t), t>0$, leaves the cone $\mathcal{M}_{+}\left(\Gamma_{0}\right)$ invariant, and $\left\|S_{\mu}(t) \mu\right\|=1$ whenever $\|\mu\|=1$. Our aim is to show that the problem (1.8) has a solution in the form

$$
\mu_{t}=S_{\mu}(t) \mu_{0}
$$

where $\left\{S_{\mu}(t)\right\}_{t \geq 0}$ is a stochastic semigroup in $\mathcal{M}\left(\Gamma_{0}\right)$, that leaves invariant important subspaces of $\mathcal{M}\left(\Gamma_{0}\right)$. For a measurable $b: \Gamma_{0} \rightarrow \mathbb{R}_{+}$, we set

$$
\mathcal{M}_{b}\left(\Gamma_{0}\right)=\left\{\mu \in \mathcal{M}\left(\Gamma_{0}\right): \mu_{ \pm}(b)<\infty\right\},
$$

where $\mu_{ \pm}$are the same as in (3.28) and

$$
\mu_{ \pm}(b):=\int_{\Gamma_{0}} b(\eta) \mu_{ \pm}(d \eta)
$$

The set $\mathcal{M}_{b}\left(\Gamma_{0}\right)$ can be equipped with the norm

$$
\|\mu\|_{b}=\alpha_{+} \mu_{+}(b)+\alpha_{-} \mu_{-}(b),
$$


which turns it into a Banach space. Set

$$
\mathcal{M}_{b,+}\left(\Gamma_{0}\right)=\mathcal{M}_{b}\left(\Gamma_{0}\right) \cap \mathcal{M}_{+}\left(\Gamma_{0}\right) .
$$

We also suppose that $b$ is such that the embedding $\mathcal{M}_{b}\left(\Gamma_{0}\right) \hookrightarrow \mathcal{M}\left(\Gamma_{0}\right)$ is dense and continuous. In the sequel, we use [32, Proposition 5.1], which we rephrase as follows.

Proposition 3.6 Suppose that $b$ and some positive $C$ and $\varepsilon$ obey the following estimate

$$
\int_{\Gamma_{0}}(b(\xi)-b(\eta)) \Omega(d \xi, \eta) \leq C b(\eta)-\varepsilon \Omega\left(\Gamma_{0}, \eta\right),
$$

which holds for all $\eta \in \Gamma_{0}$. Then the closure of $L^{*}$ as in (3.25), (3.26) with domain (3.29) generates a $C_{0}$-stochastic semigroup $\left\{S_{\mu}(t)\right\}_{t \geq 0}$, which leaves $\mathcal{M}_{b}\left(\Gamma_{0}\right)$ invariant and induces a positive $C_{0}$-semigroup on $\left(\mathcal{M}_{b}\left(\Gamma_{0}\right),\|\cdot\|_{b}\right)$.

Theorem 3.7 The problem (1.8) with a $\Gamma_{0}$-state $\mu_{0}$ has a unique classical solution in $\mathcal{M}_{+}\left(\Gamma_{0}\right)$ on $[0,+\infty)$, given by (3.30) where $S_{\mu}(t), t>0$, constitute the stochastic semigroup on $\mathcal{M}\left(\Gamma_{0}\right)$ generated by the closure of $L^{*}$ given in (3.25), (3.26), and (3.29). Moreover, for each $b$ which satisfies

$$
b(\eta)=\delta(|\eta|) \geq \varepsilon \Omega\left(\Gamma_{0}, \eta\right), \quad \text { for all } \eta \in \Gamma_{0},
$$

with some $\varepsilon>0$ and suitable $\delta: \mathbb{N} \rightarrow[0,+\infty)$, the mentioned semigroup $\left\{S_{\mu}(t)\right\}_{t \geq 0}$ leaves $\mathcal{M}_{b,+}\left(\Gamma_{0}\right)$ invariant.

Proof Computations based on (2.23) show that, for $b(\eta)=\delta(|\eta|)$, the left-hand side of (3.31) vanishes, which reflects the fact the the Kawasaki dynamics is conservative. Then the proof follows by Proposition 3.6. The condition that $\mu_{0} \in \mathcal{M}_{b,+}\left(\Gamma_{0}\right)$ with $b$ satisfying (3.32) merely means that this $\mu_{0}$ is taken from the domain of $L^{*}$, cf. (3.29).

Suppose now that the initial state $\mu_{0}$ in (1.8) is supported on $\Gamma_{0}$ and is absolutely continuous with respect to the Lebesgue-Poisson measure $\lambda$. Then

$$
R_{0}(\eta)=\frac{d \mu_{0}}{d \lambda}(\eta)
$$

is a positive element of unit norm of the Banach space $\mathcal{R}:=L^{1}\left(\Gamma_{0}, d \lambda\right)$. If $\mu_{0} \in \mathcal{M}_{b,+}\left(\Gamma_{0}\right)$, then also $R_{0} \in \mathcal{R}_{b}:=L^{1}\left(\Gamma_{0}, b d \lambda\right)$. For $b$ obeying (3.32), it is possible to show that, for any $t>0$, the solution $\mu_{t}$ as in Theorem 3.7 has the Radon-Nikodym derivative $R_{t}$ which lies in $\mathcal{R}_{b}$. Furthermore, there exists a stochastic semigroup $\left\{S_{R}(t)\right\}_{t \geq 0}$ on $\mathcal{R}$, which leaves invariant each $\mathcal{R}_{b}$ with $b$ obeying (3.32), and such that

$$
R_{t}=S_{R}(t) R_{0}, \quad t \geq 0 .
$$

The generator $L^{\dagger}$ of the semigroup $\left\{S_{R}(t)\right\}_{t \geq 0}$ has the following properties

$$
\begin{gathered}
\operatorname{Dom}\left(L^{\dagger}\right) \supset \mathcal{R}_{b}, \\
\int_{\Gamma_{0}} F(\eta)\left(L^{\dagger} R\right)(\eta) \lambda(d \eta)=\int_{\Gamma_{0}}(L F)(\eta) R(\eta) \lambda(d \eta),
\end{gathered}
$$


which holds for each $b$ obeying (3.32), and for each $R \in \mathcal{R}$ and each measurable $F: \Gamma_{0} \rightarrow \mathbb{R}$ such that both integrals in (3.36) exist. Here $L$ is as in (1.2). For each $t \geq 0$, the correlation function of $\mu_{t}$ and its Radon-Nikodym derivative satisfy, cf. (2.16),

$$
k_{\mu_{t}}(\eta)=\int_{\Gamma_{0}} R_{t}(\eta \cup \xi) \lambda(d \xi)
$$

By this representation and by (2.23), we derive

$$
\int_{\mathbb{R}^{d}} k_{\mu_{t}}^{(1)}(x) d x=\int_{\Gamma_{0}}|\eta| R_{t}(\eta) \lambda(d \eta),
$$

which yields the expected number of particles in state $\mu_{t}$. Note that we cannot expect now that $k_{\mu_{t}}$ lies in the spaces where we solve (1.9), cf. Theorem 3.1.

\subsection{The Evolution of States}

Recall that by $B_{\mathrm{bs}}\left(\Gamma_{0}\right)$ we denote the set of all bounded measurable functions $G: \Gamma_{0} \rightarrow \mathbb{R}$ each of which is supported on a bounded $A$, cf. (2.6). Its subset $B_{\mathrm{bs}}^{+}\left(\Gamma_{0}\right)$ is defined in (2.17).

Given $\vartheta \in \mathbb{R}$, let $\mathcal{M}_{\vartheta}(\Gamma)$ stand for the set of all $\mu \in \mathcal{M}_{\mathrm{fm}}^{1}(\Gamma)$, for which $k_{\mu} \in \mathcal{K}_{\vartheta}$, see (2.16) and (3.10). Let also $\mathcal{K}_{\vartheta}^{+}$be the set of all $k \in \mathcal{K}_{\vartheta}$ such that, cf. (2.18),

$$
\int_{\Gamma_{0}} G(\eta) k(\eta) \lambda(d \eta) \geq 0
$$

which holds for every $G \in B_{\mathrm{bs}}^{+}\left(\Gamma_{0}\right)$. Note that this property is 'more than the mere positivity' as $B_{\mathrm{bs}}^{+}\left(\Gamma_{0}\right)$ can contain functions which take also negative values, see (2.13) and (2.17). Then in view of Proposition 2.2, the map $\mathcal{M}_{\vartheta}(\Gamma) \ni \mu \mapsto k_{\mu} \in \mathcal{K}_{\vartheta}^{+}$is a bijection as such $k_{\mu}$ certainly obeys (2.19). In what follows, the evolution of states $\mu_{0} \mapsto \mu_{t}$ is understood as the evolution of the corresponding correlation functions $k_{\mu_{0}} \mapsto k_{\mu_{t}}$ obtained by solving the problem (1.9).

Theorem 3.8 Let $\vartheta_{0} \in \mathbb{R}, \vartheta$, and $T(\vartheta)$ be as in Theorem 3.1 and in (3.13), respectively, and let $\mu_{0}$ be in $\mathcal{M}_{\vartheta_{0}}(\Gamma)$. Then the evolution described in Theorem 3.1 with $k_{0}=k_{\mu_{0}}$ leaves $\mathcal{K}_{\vartheta}^{+}$ invariant, which means that each $k_{t}$ is the correlation function of a unique $\mu_{t} \in \mathcal{M}_{\vartheta}(\Gamma)$. Hence, the evolution $k_{\mu_{0}} \mapsto k_{t}, t \in[0, T(\vartheta))$, determines the evolution of states

$$
\mathcal{M}_{\vartheta_{0}}(\Gamma) \ni \mu_{0} \mapsto \mu_{t} \in \mathcal{M}_{\vartheta}(\Gamma), \quad t \in[0, T(\vartheta))
$$

Proof To prove the statement we have to show that a solution $k_{t}$ of the problem (1.9) with $k_{0}=k_{\mu_{0}}$ obeys (3.38) for all $t \in(0, T(\vartheta))$. Fix $\mu_{0} \in \mathcal{M}_{\vartheta_{0}}(\Gamma)$ and take $\Lambda \in \mathcal{B}_{\mathrm{b}}\left(\mathbb{R}^{d}\right)$. Let $\mu_{0}^{\Lambda}$ be the projection of $\mu_{0}$ onto $\Gamma_{\Lambda}$, cf. (2.14). Since $\mu_{0}$ is in $\mathcal{M}_{\mathrm{fm}}^{1}(\Gamma)$, its density $R_{0}^{\Lambda}$, as in (3.33), is in $\mathcal{R}$. Given $N \in \mathbb{N}$, we let $I_{N}(\eta)=1$ whenever $|\eta| \leq N$, and $I_{N}(\eta)=0$ otherwise. Then we set

$$
R_{0}^{\Lambda, N}(\eta)=R_{0}^{\Lambda}(\eta) I_{N}(\eta)
$$

As a function on $\Gamma_{0}, R_{0}^{\Lambda, N}$ is a collection of $R_{0}^{\Lambda, N, n}:\left(\mathbb{R}^{d}\right)^{n} \rightarrow \mathbb{R}_{+}, n \in \mathbb{N}_{0}$. Clearly, $R_{0}^{\Lambda, N}$ is a positive element of $\mathcal{R}$ of norm $\left\|R_{0}^{\Lambda, N}\right\|_{\mathcal{R}} \leq 1$. Furthermore, for each $\beta>0$,

$$
\int_{\Gamma_{0}} e^{\beta|\eta|} R_{0}^{\Lambda, N}(\eta) \lambda(d \eta)=\sum_{n=0}^{N} \frac{e^{n \beta}}{n !} \int_{\Lambda^{n}} R_{0}^{\Lambda, N, n}\left(x_{1}, \ldots, x_{n}\right) d x_{1} \cdots d x_{n}<\infty,
$$


and hence $R_{0}^{\Lambda, N} \in \mathcal{R}_{b}$ with $b(\eta)=e^{\beta|\eta|}$, for each $\beta>0$. Set

$$
R_{t}^{\Lambda, N}=S_{R}(t) R_{0}^{\Lambda, N} . t \geq 0,
$$

where $S_{R}(t)$ is as in (3.34). By Theorem 3.7, we have that

$$
\begin{aligned}
\forall t \geq 0: & \text { (a) } \forall \beta>0 \quad R_{t}^{\Lambda, N} \in \mathcal{R}_{b}, \quad \text { with } b(\eta)=e^{\beta|\eta|}, \\
& \text { (b) } R^{\Lambda, N}(\eta) \geq 0, \text { for } \lambda \text { - a.a. } \eta \in \Gamma_{0}, \\
& \text { (c) }\left\|R_{t}^{\Lambda, N}\right\|_{\mathcal{R}} \leq 1 .
\end{aligned}
$$

Furthermore, in view of (3.35), by [28, Theorem 2.4, pp. 4-5] we have from (3.40)

$$
R_{t}^{\Lambda, N}=R_{0}^{\Lambda, N}+\int_{0}^{t} L^{\dagger} R_{s}^{\Lambda, N} d s .
$$

Set, cf. (2.16) and (3.37),

$$
q_{t}^{\Lambda, N}(\eta)=\int_{\Gamma_{0}} R_{t}^{\Lambda, N}(\eta \cup \xi) \lambda(d \xi) .
$$

For $G \in B_{\mathrm{bs}}\left(\Gamma_{0}\right)$, let $N(G) \in \mathbb{N}_{0}$ be such that $G^{(n)} \equiv 0$ for $n>N(G)$. For such $G, K G$ is a cylinder function on $\Gamma$, which can also be considered as a measurable function on $\Gamma_{0}$. By (2.13), we have that, for every $G \in B_{\mathrm{bs}}\left(\Gamma_{0}\right)$ and each $t \geq 0$,

$$
\left\langle\left\langle K G, R_{t}^{\Lambda, N}\right\rangle=\left\langle\left\langle G, q_{t}^{\Lambda, N}\right\rangle,\right.\right.
$$

see (3.4). Since $G \in B_{\mathrm{bs}}\left(\Gamma_{0}\right)$ is bounded, we have

$$
C(G):=\max _{n \in\{0, \ldots, N(G)\}}\left\|G^{(n)}\right\|_{L^{\infty}\left(\left(\mathbb{R}^{d}\right)^{n}\right)}<\infty,
$$

which immediately yields that

$$
|(K G)(\eta)| \leq(1+|\eta|)^{N(G)} C(G),
$$

and hence both integrals in (3.44) exist since $R_{t}^{\Lambda, N} \in \mathcal{R}_{b}$ for $b(\eta)=e^{\beta|\eta|}$ with any $\beta>0$. Moreover, by the same argument the map $\mathcal{R} \ni R \mapsto\langle\langle K G, R\rangle$ is continuous, and thus from (3.42) and (3.36) we obtain

$$
\begin{aligned}
\left\langle\left\langle K G, R_{t}^{\Lambda, N}\right\rangle\right. & =\left\langle\left\langle K G, R_{0}^{\Lambda, N}\right\rangle+\int_{0}^{t} \|\left\langle K G, L^{\dagger} R_{s}^{\Lambda, N}\right\rangle d s\right. \\
& =\left\langle\left\langle K G, R_{0}^{\Lambda, N}\right\rangle+\int_{0}^{t}\left\langle\left\langle L K G, R_{s}^{\Lambda, N}\right\rangle d s .\right.\right.
\end{aligned}
$$

Now we would want to interchange in the latter line $L$ and $K$. If $\widehat{L} G$ were in $B_{\mathrm{bs}}\left(\Gamma_{0}\right)$, one could get point-wise $L K G=K \widehat{L} G-$ by the very definition of $\widehat{L}$. However, this is not the case since, $\mathrm{cf}$. (3.7),

$$
|(\widehat{L} G)(\eta)| \leq(K U G)(\eta)
$$


where

$$
(U G)(\xi)=\sum_{x \in \xi} \int_{\mathbb{R}^{d}} a(x-y)|G(\xi \backslash x \cup y)-G(\xi)| d y .
$$

Here we used, cf. (2.11) and (3.2), that

$$
0 \leq e\left(\tau_{y}, \xi\right) \leq 1, \quad\left|e\left(t_{y}, \eta \backslash \xi\right)\right| \leq 1,
$$

which holds for almost all all $y, \xi$, and $\eta$. Then, for $G \in B_{\mathrm{bs}}\left(\Gamma_{0}\right)$, we have, $\mathrm{cf}$ (3.45),

$$
N(U G)=N(G), \quad C(U G) \leq 2 \alpha N(G) C(G),
$$

which then yields

$$
|(\widehat{L} G)(\eta)| \leq 2 \alpha N(G) C(G)(1+|\eta|)^{N(G)} .
$$

Let us show that, for any $t \geq 0$, the function $(\widehat{L} G) q_{t}^{\Lambda, N}$ is $\lambda$-integrable, cf. (3.43). By (2.23), from (3.43) and (3.47) we get

$$
\begin{aligned}
\left.\| \widehat{L} G, q_{t}^{\Lambda, N}\right\rangle & \leq 2 \alpha N(G) C(G) \int_{\Gamma_{0}} R_{t}^{\Lambda, N}(\eta)\left(\sum_{\xi \subset \eta}(1+|\xi|)^{N(G)}\right) \lambda(d \eta) \\
& \leq 2 \alpha N(G) C(G) \int_{\Gamma_{0}} 2^{|\eta|}(1+|\eta|)^{N(G)} R_{t}^{\Lambda, N}(\eta) \lambda(d \eta) .
\end{aligned}
$$

Hence, by claim $(a)$ in (3.41) we get the integrability in question. Then by (3.44) we transform (3.46) into

$$
\|\left\langle G, q_{t}^{\Lambda, N}\right\rangle=\left\langle\left\langle G, q_{0}^{\Lambda, N}\right\rangle+\int_{0}^{t} \| \widehat{L} G, q_{s}^{\Lambda, N}\right\rangle d s .
$$

Since $R_{t}^{\Lambda, N}$ is positive, cf. (b) in (3.41), by (3.44) we get

$$
\|\left\langle G, q_{t}^{\Lambda, N}\right\rangle \geq 0 \quad \text { for } \quad G \in B_{\mathrm{bs}}^{+}\left(\Gamma_{0}\right) .
$$

On the other hand, by (3.39) and (3.43) we have, see also (2.16),

$$
0 \leq q_{0}^{\Lambda, N}(\eta) \leq \int_{\Gamma_{0}} R^{\Lambda}(\eta \cup \xi) \lambda(d \xi)=k_{\mu_{0}}(\eta) \mathbb{I}_{\Gamma_{\Lambda}}(\eta) \leq k_{\mu_{0}}(\eta),
$$

where $\mathbb{I}_{\Gamma_{\Lambda}}$ is the indicator of $\Gamma_{\Lambda}$, i.e., $\mathbb{I}_{\Gamma_{\Lambda}}(\eta)=1$ whenever $\eta \in \Gamma_{\Lambda}$, and $\mathbb{I}_{\Gamma_{\Lambda}}(\eta)=0$ otherwise. By (3.50), $q_{0}^{\Lambda, N} \in \mathcal{K}_{\vartheta_{0}}$. Let $k_{t}^{\Lambda, N}, t \in[0, T)$, be the solution of (1.9) with $k_{0}=q_{0}^{\Lambda, N}$, as stated in Theorem 3.1. Then

$$
k_{t}^{\Lambda, N}=k_{0}^{\Lambda, N}+\int_{0}^{t} L^{\Delta} k_{s}^{\Lambda, N} d s,
$$

which for $G$ as in (3.48) yields

$$
\left\langle\left\langle G, k_{t}^{\Lambda, N}\right\rangle=\left\|\left\langle G, q_{0}^{\Lambda, N}\right\rangle+\int_{0}^{t}\right\| \widehat{L} G, k_{s}^{\Lambda, N}\right\rangle d s .
$$


Set

$$
\varphi(t ; G)=\left\langle\left\langle G, q_{t}^{\Lambda, N}\right\rangle, \quad \psi(t ; G)=\left\langle\left\langle G, k_{t}^{\Lambda, N} \| .\right.\right.\right.
$$

By (3.48) and (3.51), we obtain, cf. Corollary 3.5,

$$
\frac{d^{n} \varphi}{d t^{n}}(0 ; G)=\frac{d^{n} \psi}{d t^{n}}(0 ; G)=\left\langle\left\langle\widehat{L}^{n} G, q_{0}^{\Lambda, N}\right\rangle=\left\langle\left\langle G,\left(L^{\Delta}\right)^{n} q_{0}^{\Lambda, N}\right\rangle .\right.\right.
$$

From this we can get that, cf. (3.49),

$$
\left.\| G, k_{t}^{\Lambda, N}\right\rangle=\left\langle\left\langle G, q_{t}^{\Lambda, N}\right\rangle \geq 0, \quad \text { for } \quad G \in B_{\mathrm{bs}}^{+}\left(\Gamma_{0}\right),\right.
$$

provided the series

$$
\sum_{m=0}^{\infty} \frac{t^{m}}{m !}\left\langle\left\langle G,\left(L^{\Delta}\right)^{m} q_{0}^{\Lambda, N}\right\rangle\right.
$$

converges for all $t \in[0, T(\vartheta))$, cf. (3.52). But the latter indeed holds true in view of (3.21), which implies that (3.53) holds for all $t \in[0, T(\vartheta))$.

In Appendix, we show that, for each $G \in B_{\mathrm{bs}}^{+}\left(\Gamma_{0}\right)$ and any $t \in[0, T(\vartheta))$,

$$
\left\langle\left\langle G, k_{t}\right\rangle\right\rangle=\lim _{n \rightarrow+\infty} \lim _{l \rightarrow+\infty}\left\langle\left\langle G, k_{t}^{\Lambda_{n}, N_{l}}\right\rangle\right\rangle,
$$

for certain increasing sequences $\left\{\Lambda_{n}\right\}_{n \in \mathbb{N}}$ and $\left\{N_{l}\right\}_{l \in \mathbb{N}}$ such that $N_{l} \rightarrow+\infty$ and $\Lambda_{n} \rightarrow \mathbb{R}^{d}$. Then by (3.54) and (3.53) we obtain (3.38), and thus complete the proof.

\section{Mesoscopic Dynamics}

As mentioned above, the mesoscopic description of the considered model is obtained by means of a Vlasov-type scaling, originally developed for describing mesoscopic properties of plasma. We refer to [7,29,31] as to the source of general concepts in this field, as well as to [12] where the peculiarities of the scaling method which we use are given along with the updated bibliography on this item.

\subsection{The Vlasov Hierarchy}

The main idea of the scaling which we use in this article is to make the particle system more and more dense whereas the interaction respectively weaker. This corresponds to the so called mean field approximation widely employed in theoretical physics. Note that we are not scaling time, which would be the case for a macroscopic scaling. The object of our manipulations will be the problem (1.9). The scaling parameter $\varepsilon>0$ will be tending to zero. The first step is to assume that the initial state depends on $\varepsilon$ in such a way that the correlation function $k_{0}^{(\varepsilon)}$ diverges as $\varepsilon \rightarrow 0$ in such a way that the so called renormalized correlation function

$$
k_{0, \text { ren }}^{(\varepsilon)}(\eta):=\varepsilon^{|\eta|} k_{0}^{(\varepsilon)}
$$

converges $k_{0, \text { ren }}^{(\varepsilon)} \rightarrow r_{0}$, as $\varepsilon \rightarrow 0$, to the correlation function of a certain measure. Let $k_{0}^{(\varepsilon, n)}:\left(\mathbb{R}^{d}\right)^{n} \rightarrow \mathbb{R}$ denote $n$-particle 'component' of $k_{0}^{(\varepsilon)}$. Then our assumption, in particular, 
means

$$
k_{0}^{(\varepsilon, 1)} \sim \varepsilon^{-1} .
$$

Then the second step is to consider the Cauchy problem

$$
\frac{d}{d t} k_{t}^{(\varepsilon)}=L_{\varepsilon}^{\Delta} k_{t}^{(\varepsilon)},\left.\quad k_{t}^{(\varepsilon)}\right|_{t=0}=k_{0}^{(\varepsilon)}
$$

where $L_{\varepsilon}^{\Delta}$ is as in (3.1) but with $\phi$ multiplied by $\varepsilon$. As might be seen from (3.19), the solution $k_{t}^{(\varepsilon)}$, which exists in view of Theorem 3.1, diverges as $\varepsilon \rightarrow 0$. Thus, similarly as in (4.1) we pass to

$$
k_{t, \mathrm{ren}}^{(\varepsilon)}(\eta)=\varepsilon^{|\eta|} k_{t}^{(\varepsilon)},
$$

which means that instead of (4.3) we are going to solve the following problem

$$
\frac{d}{d t} k_{t, \text { ren }}^{(\varepsilon)}=\left.L_{\varepsilon, \text { ren }} k_{t, \text { ren }}^{(\varepsilon)} \quad k_{t, \text { ren }}^{(\varepsilon)}\right|_{t=0}=k_{0, \text { ren }}^{(\varepsilon)},
$$

with

$$
L_{\varepsilon, \text { ren }}=R_{\varepsilon}^{-1} L_{\varepsilon}^{\Delta} R_{\varepsilon}, \quad\left(R_{\varepsilon} k\right)(\eta):=\varepsilon^{-|\eta|} k(\eta) .
$$

Remark 4.1 Since $k_{0}^{(\varepsilon)}$ is a correlation function, by Theorem 3.7 we know that $k_{t}^{(\varepsilon)}$ is the correlation function of a unique measure $\mu_{t}^{(\varepsilon)}$. If $\mu_{0}^{(1)}$ is a Poisson measure with density $k_{0}^{(1,1)}=\varrho_{0}$, then also $\mu_{0}^{(\varepsilon)}$ with density $k_{0}^{(\varepsilon, 1)}=\varepsilon^{-1} \varrho_{0}$ is a Poisson measure. We can expect that, for $t>0, k_{t, \text { ren }}^{(\varepsilon)}$ has a nontrivial limit as $\varepsilon \rightarrow 0^{+}$, only if $k_{t}^{(\varepsilon)}(\eta) \leq\left[k_{t}^{(\varepsilon, 1)}(x)\right]^{|\eta|}$, cf. (4.2) and (4.4). For this to hold, $\mu_{t}^{(\varepsilon)}$ should be sub-Poissonian, cf. Definition 2.3 and Remark 2.4. That is, the evolution $\mu_{0}^{(\varepsilon)} \mapsto \mu_{t}^{(\varepsilon)}$ should preserve sub-Poissonicity, which is the case by Theorem 3.1 in view of (3.8).

By (3.1) and (4.6), we have

$$
\begin{aligned}
\left(L_{\varepsilon, \text { ren }}\right)(\eta)=\sum_{y \in \eta} \int_{\mathbb{R}^{d}} a(x-y) e\left(\tau_{y}^{(\varepsilon)}, \eta \backslash y \cup x\right) & \\
& \times\left(\int_{\Gamma_{0}} e\left(\varepsilon^{-1} t_{y}^{(\varepsilon)}, \xi\right) k(\xi \cup x \cup \eta \backslash y) \lambda(d \xi)\right) d x \\
& -\int_{\Gamma_{0}} k(\xi \cup \eta)\left(\sum_{x \in \eta} \int_{\mathbb{R}^{d}} a(x-y) e\left(\tau_{y}^{(\varepsilon)}, \eta\right)\right. \\
& \left.\times e\left(\varepsilon^{-1} t_{y}^{(\varepsilon)}, \xi\right) d y\right) \lambda(d \xi),
\end{aligned}
$$

where, cf. (3.2),

$$
t_{x}^{(\varepsilon)}(y)=e^{-\varepsilon \phi(x-y)}-1, \quad \tau_{x}^{(\varepsilon)}(y)=t_{x}^{(\varepsilon)}(y)+1 .
$$

As in (3.18), for any $\vartheta^{\prime} \in \mathbb{R}$ and $\vartheta^{\prime \prime}>\vartheta^{\prime}$, we have

$$
\left\|L_{\varepsilon, \operatorname{ren}}\right\|_{\vartheta^{\prime \prime} \vartheta^{\prime}} \leq \frac{2 \alpha}{e\left(\vartheta^{\prime \prime}-\vartheta^{\prime}\right)} \exp \left(c_{\phi}^{(\varepsilon)} e^{-\vartheta^{\prime \prime}}\right),
$$


where, cf. (1.6),

$$
c_{\phi}^{(\varepsilon)}=\varepsilon^{-1} \int_{\mathbb{R}^{d}}\left(1-e^{-\varepsilon \phi(x)}\right) d x .
$$

Suppose now that $\phi$ is in $L^{1}\left(\mathbb{R}^{d}\right)$ and set

$$
\langle\phi\rangle=\int_{\mathbb{R}^{d}} \phi(x) d x .
$$

Recall that we still assume $\phi \geq 0$. Then

$$
\left\|L_{\varepsilon, \text { ren }}\right\|_{\vartheta^{\prime \prime} \vartheta^{\prime}} \leq \sup _{\varepsilon>0}\{\operatorname{RHS}(4.8)\}=\frac{2 \alpha}{e\left(\vartheta^{\prime \prime}-\vartheta^{\prime}\right)} \exp \left(\langle\phi\rangle e^{-\vartheta^{\prime \prime}}\right) .
$$

Let us now, informally, pass in (4.7) to the limit $\varepsilon \rightarrow 0$. Then we get the following operator

$$
\begin{aligned}
\left(L_{V} k\right)(\eta)= & \sum_{y \in \eta} \int_{\mathbb{R}^{d}} a(x-y) \int_{\Gamma_{0}} e(-\phi(y-\cdot), \xi) \\
& \times k(\xi \cup x \cup \eta \backslash y) \lambda(d \xi) d x \\
& -\int_{\Gamma_{0}} k(\xi \cup \eta) \sum_{x \in \eta} \int_{\mathbb{R}^{d}} a(x-y) \\
& \times e(-\phi(y-\cdot), \xi) d y \lambda(d \xi) .
\end{aligned}
$$

It certainly obeys

$$
\left\|L_{V}\right\|_{\vartheta^{\prime \prime} \vartheta^{\prime}} \leq \frac{2 \alpha}{e\left(\vartheta^{\prime \prime}-\vartheta^{\prime}\right)} \exp \left(\langle\phi\rangle e^{-\vartheta^{\prime \prime}}\right),
$$

and hence along with (4.3) we can consider the problem

$$
\frac{d}{d t} r_{t}=L_{V} r_{t},\left.\quad r_{t}\right|_{t=0}=r_{0}
$$

which is called the Vlasov hierarchy for the Kawasaki system which we consider. Repeating the arguments used in the proof of Theorem 3.1 we obtain the following

Proposition 4.2 For every $\vartheta_{0} \in \mathbb{R}$, there exists $T_{*}=T_{*}\left(\vartheta_{0}, \alpha,\langle\phi\rangle\right)$ such that the problem (4.5) (resp. (4.11)) with any $\varepsilon>0$ and $k_{0}^{(\varepsilon)} \in \mathcal{K}_{\vartheta_{0}}$ (resp. $r_{0} \in \mathcal{K}_{\vartheta_{0}}$ ) has a unique classical solution $k_{t}^{(\varepsilon)} \in \mathcal{K}_{\vartheta(t)}\left(\right.$ resp. $\left.r_{t} \in \mathcal{K}_{\vartheta(t)}\right)$ for $t \in\left[0, T_{*}\right)$.

As mentioned in Remark 4.1, $k_{t}^{(\varepsilon)}$ is also a correlation function if $k_{0}^{(\varepsilon)}$ is so. However, this could not be the case for $r_{t}$, even if $r_{0}=k_{0}^{(\varepsilon)}$. Moreover, we do not know how 'close' is $r_{t}$ to $k_{t}^{(\varepsilon)}$, as the passage from $L_{\varepsilon}$, ren to $L_{V}$ was only informal. In the remaining part of the article we give answers to both these questions.

\subsection{The Vlasov Equation}

Here we show that the problem (4.12) has a very particular solution, which gives sense to the whole construction. For $a$ as in (1.3) and an appropriate $g: \mathbb{R}^{d} \rightarrow \mathbb{R}$, we write 


$$
(a * g)(x)=\int_{\mathbb{R}^{d}} a(x-y) g(y) d y,
$$

and similarly for $\phi * g$. Then let us consider in $L^{\infty}\left(\mathbb{R}^{d}\right)$ the following Cauchy problem

$$
\begin{aligned}
\frac{d}{d t} \varrho_{t}(x)= & \left(a * \varrho_{t}\right)(x) \exp \left[-\left(\varrho_{t} * \phi\right)(x)\right] \\
& -\varrho_{t}(x)\left(a * \exp \left(-\varrho_{t} * \phi\right)\right)(x), \\
\left.\varrho_{t}\right|_{t=0}= & \varrho_{0} .
\end{aligned}
$$

Denote

$$
\begin{aligned}
& \Delta^{+}=\left\{\varrho \in L^{\infty}\left(\mathbb{R}^{d}\right): \varrho(x) \geq 0 \text { for a.a. } x\right\}, \\
& \Delta_{u}=\left\{\varrho \in L^{\infty}\left(\mathbb{R}^{d}\right):\|\varrho\|_{L^{\infty}\left(\mathbb{R}^{d}\right)} \leq u\right\}, \quad u>0, \\
& \Delta_{u}^{+}=\Delta^{+} \cap \Delta_{u} .
\end{aligned}
$$

Lemma 4.3 Let $\vartheta_{0}$ and $T_{*}$ be as in Proposition 4.2. Suppose that, for some $T \in\left(0, T_{*}\right)$, the problem (4.13) with $\varrho_{0} \in \Delta_{u_{0}}^{+}$, has a unique classical solution $\varrho_{t} \in \Delta_{u_{T}}^{+}$on $[0, T]$, for some $u_{T}>0$. Then, for $\vartheta_{0}=-\log u_{0}$ and $\vartheta(T)=-\log u_{T}$, the solution $r_{t} \in \mathcal{K}_{\vartheta(T)}$ of (4.11) as in Proposition 4.2 with $r_{0}(\eta)=e\left(\varrho_{0}, \eta\right)$ is given by

$$
r_{t}(\eta)=e\left(\varrho_{t}, \eta\right)=\prod_{x \in \eta} \varrho_{t}(x)
$$

Proof First of all we note that, for a given $\vartheta, e(\varrho, \cdot) \in \mathcal{K}_{\vartheta}$ if and only if $\varrho \in \Delta_{u}$ with $u=e^{-\vartheta}$, see (3.8). Now set $\tilde{r}_{t}=e\left(\varrho_{t}, \cdot\right)$ with $\varrho_{t}$ solving (4.13). This $\tilde{r}_{t}$ solves (4.12), which can easily be checked by computing $d / d t$ and employing (4.13). In view of the uniqueness as in Proposition 4.2, we then have $\tilde{r}_{t}=r_{t}$ on $[0, T]$, from which it can be continued to $\left[0, T_{*}\right)$.

Remark 4.4 As (4.14) is the correlation function of the Poisson measure $\pi_{\varrho_{t}}$, see (2.9) and (2.10), the above lemma establishes the so called chaos preservation or chaos propagation in time. Indeed, the most chaotic states those corresponding to Poisson measures, cf. (2.20), (2.21), and (2.22).

Let us show now that the problem (4.13) does have the solution we need. In a standard way, (4.13) can be transformed into the following integral equation

$$
\begin{aligned}
\varrho_{t}(x)= & F_{t}(\varrho)(x):=\varrho_{0}(x) e^{-\alpha t} \\
& +\int_{0}^{t} \exp (-\alpha(t-s))\left(a * \varrho_{s}\right)(x) \exp \left[-\left(\varrho_{s} * \phi\right)(x)\right] d s \\
& +\int_{0}^{t} \exp (-\alpha(t-s)) \varrho_{s}(x)\left[a *\left(1-\exp \left(-\varrho_{s} * \phi\right)\right)\right](x) d s,
\end{aligned}
$$

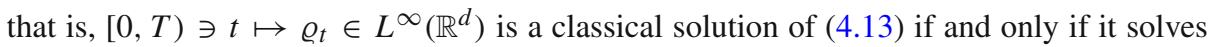
(4.15). Suppose $\varrho_{t} \in \Delta^{+}$is such a solution. Then we set

$$
u_{t}=\left\|\varrho_{t}\right\|_{L^{\infty}\left(\mathbb{R}^{d}\right)}, \quad t \in[0, T) .
$$


Since $\phi \geq 0$ and $\varrho_{t} \in \Delta^{+}$, from (4.15) we get for $v_{t}:=u_{t} \exp (\alpha t)$, cf. (1.4),

$$
v_{t} \leq v_{0}+2 \alpha \int_{0}^{t} v_{s} d s,
$$

from which by the Gronwall inequality we obtain $v_{t} \leq v_{0} \exp (2 \alpha t)$; and hence,

$$
u_{t} \leq u_{0} e^{\alpha t} .
$$

In a similar way, one shows that, for $\varrho_{0} \in \Delta_{u_{0}}^{+}$and $\varrho_{s} \in \Delta_{u_{t}}^{+}$for all $s \in[0, t]$,

$$
F_{t}(\varrho) \in \Delta_{u_{t}}^{+}, \quad u_{t}:=\frac{u_{0}}{2-e^{\alpha t}} .
$$

Now for $\varrho_{0} \in \Delta_{u_{0}}^{+}$and some $t>0$ such that $e^{\alpha t}<2$, cf. (4.18), we consider the sequence

$$
\varrho_{t}^{(0)}=\varrho_{0}, \quad \varrho_{t}^{(n)}=F_{t}\left(\varrho^{(n-1)}\right), \quad n \in \mathbb{N} .
$$

Obviously, each $\varrho_{t}^{(n)}$ is in $\Delta_{u_{t}}^{+}$. Now let us find $T<\min \left\{T_{*}, \log 2 / \alpha\right\}, T_{*}$ being as in Lemma 4.3 , such that the sequence of

$$
\delta_{n}:=\sup _{t \in[0, T]}\left\|\varrho_{t}^{(n)}-\varrho_{t}^{(n-1)}\right\|_{L^{\infty}\left(\mathbb{R}^{d}\right)}, \quad n \in \mathbb{N}
$$

is summable, which would guarantee that, for each $t \leq T,\left\{\varrho_{t}^{(n)}\right\}_{n \in \mathbb{N}_{0}}$ is a Cauchy sequence. For $\varrho_{s}^{(n-1)}, \varrho_{s}^{(n-2)} \in \Delta_{u_{T}}$, we have

$$
\begin{gathered}
\left\|1-\exp \left(\phi *\left(\varrho_{s}^{(n-1)}-\varrho_{s}^{(n-2)}\right)\right)\right\| L^{\infty}\left(\mathbb{R}^{d}\right) \leq\left\|\phi *\left(\varrho_{s}^{(n-1)}-\varrho_{s}^{(n-2)}\right)\right\| L^{\infty}\left(\mathbb{R}^{d}\right) \\
\quad \times \sum_{m=0}^{\infty} \frac{1}{m !} \frac{1}{m+1}\left\|\phi *\left(\varrho_{s}^{(n-1)}-\varrho_{s}^{(n-2)}\right)\right\| \begin{array}{l}
m \\
L^{\infty}\left(\mathbb{R}^{d}\right)
\end{array} \\
\leq\langle\phi\rangle\left\|\varrho_{s}^{(n-1)}-\varrho_{s}^{(n-2)}\right\|_{L^{\infty}\left(\mathbb{R}^{d}\right)} \exp \left(2\langle\phi\rangle u_{T}\right) .
\end{gathered}
$$

By means of this estimate, we obtain from (4.15) and (4.19)

$$
\delta_{n} \leq q(T) \delta_{n-1},
$$

where

$$
q(T)=2\left(1-e^{-\alpha T}\right)\left(1+\langle\phi\rangle u_{0} \exp \left(\alpha T+2\langle\phi\rangle u_{0} e^{\alpha T}\right)\right) .
$$

Since $q(T)$ is a continuous increasing function such that $q(0)=0$, one finds $T>0$ such that $q(T)<1$. For this $T$, the sequence $\left\{\varrho_{t}^{(n)}\right\}_{n \in \mathbb{N}_{0}}$ converges to some $\varrho_{t} \in \Delta_{u_{T}}^{+}$, uniformly on $[0, T]$. Clearly, this $\varrho_{t}$ solves (4.13) and hence (4.15).

Theorem 4.5 The unique classical solution of (4.12) with $r_{0}=e\left(\varrho_{0}, \cdot\right), \varrho_{0} \in \Delta^{+}$, exists for all $t>0$ and is given by (4.14) with $\varrho_{t} \in \Delta^{+}$being the solution of (4.13). Moreover, this solution obeys

$$
r_{t}(\eta) \leq r_{0}(\eta) \exp (\alpha|\eta| t) .
$$

Proof For a given $\varrho_{0} \in \Delta^{+}$, we find $T$ such that $q(T)<1$, cf. (4.16) and (4.20). Then there exists a unique classical solution of (4.13) $\varrho_{t} \in \Delta_{u_{T}}^{+}$on $[0, T]$, which by Lemma 4.3 yields the solution (4.14). Since $\varrho_{t}$ obeys the a priori bound (4.17), it does not explode and hence can be continued, which yields also the continuation of $r_{t}$. Finally, the bound (4.21) follows from (4.17). 


\subsection{The Scaling Limit $\varepsilon \rightarrow 0$}

Our final task in this work is to show that the solution of (4.5) $k_{t, \text { ren }}^{(\varepsilon)}$ converges in $\mathcal{K}_{\vartheta}$ uniformly on compact subsets of $\left[0, T_{*}\right)$ to that of (4.12), see Proposition 4.2. Here we should impose an additional condition on the potential $\phi$, which, however, seems quite natural. Recall that in this section we suppose $\phi \in L^{1}\left(\mathbb{R}^{d}\right)$.

Theorem 4.6 Let $\vartheta_{0}$ and $T_{*}$ be as in Proposition 4.2, and for $T \in\left[0, T_{*}\right)$, take $\vartheta$ such that $T<T(\vartheta)$, see (3.13). Assume also that $\phi \in L^{1}\left(\mathbb{R}^{d}\right) \cap L^{\infty}\left(\mathbb{R}^{d}\right)$ and consider the problems (4.5) and (4.12) with $k_{0, \text { ren }}^{(\varepsilon)}=r_{0} \in \mathcal{K}_{\vartheta_{0}}$. For their solutions $k_{t, \text { ren }}^{(\varepsilon)}$ and $r_{t}$, it follows that $k_{t, \text { ren }}^{(\varepsilon)} \rightarrow r_{t}$ in $\mathcal{K}_{\vartheta}$, as $\varepsilon \rightarrow 0$, uniformly on $[0, T]$.

Proof For $n \in \mathbb{N}$, let $k_{t, n}^{(\varepsilon)}$ and $r_{t, n}$ be defined as in (3.19) with $L_{\varepsilon, \text { ren }}$ and $L_{V}$, respectively. As in the proof of Theorem 3.1, one can show that the sequences of $k_{t, n}^{(\varepsilon)}$ and $r_{t, n}$ converge in $\mathcal{K}_{\vartheta}$ to $k_{t, \text { ren }}^{(\varepsilon)}$ and $r_{t}$, respectively, uniformly on $[0, T]$. Then, for $\delta>0$, one finds $n \in \mathbb{N}$ such that, for all $t \in[0, T]$,

$$
\left\|k_{t, n}^{(\varepsilon)}-k_{t, \text { ren }}^{(\varepsilon)}\right\|_{\vartheta}+\left\|r_{t, n}-r_{t}\right\|_{\vartheta}<\delta / 2 .
$$

From (3.19) we then have

$$
\begin{aligned}
\left\|k_{t, \text { ren }}^{(\varepsilon)}-r_{t}\right\|_{\vartheta} & \leq\left\|\sum_{m=1}^{n} \frac{1}{m !} t^{m}\left(L_{\varepsilon, \text { ren }}^{m}-L_{V}^{m}\right) r_{0}\right\| \vartheta+\frac{\delta}{2} \\
& \leq\left\|L_{\varepsilon, \text { ren }}-L_{V}\right\|_{\vartheta_{0} \vartheta}\left\|r_{0}\right\|_{\vartheta_{0}} T \exp (T b(\vartheta))+\frac{\delta}{2},
\end{aligned}
$$

where, see (4.9) and (4.11),

$$
b(\vartheta):=\frac{2 \alpha}{e(\vartheta 0-\vartheta)} \exp \left(\langle\phi\rangle e^{-\vartheta}\right) .
$$

Here we used the following identity

$$
\begin{gathered}
L_{\varepsilon, \text { ren }}^{m}-L_{V}^{m}=\left(L_{\varepsilon, \text { ren }}-L_{V}\right) L_{\varepsilon, \text { ren }}^{m-1}+L_{V}\left(L_{\varepsilon, \text { ren }}-L_{V}\right) L_{\varepsilon, \text { ren }}^{m-2} \\
+\cdots+L_{V}^{m-2}\left(L_{\varepsilon, \text { ren }}-L_{V}\right) L_{\varepsilon, \text { ren }}+L_{V}^{m-1}\left(L_{\varepsilon, \text { ren }}-L_{V}\right) .
\end{gathered}
$$

Thus, we have to show that

$$
\left\|L_{\varepsilon, \text { ren }}-L_{V}\right\|_{\vartheta_{0} \vartheta} \rightarrow 0, \quad \text { as } \varepsilon \rightarrow 0,
$$

which will allow us to make the first summand in the right-hand side of (4.22) also smaller than $\delta / 2$ and thereby to complete the proof.

Subtracting (4.10) from (4.7) we get

$$
\begin{aligned}
\left(L_{\varepsilon, \text { ren }}-L_{V}\right) k(\eta)=\sum_{y \in \eta} & \int_{\mathbb{R}^{d}} \int_{\Gamma_{0}} a(x-y) k(\xi \cup x \cup \eta \backslash y) \\
& \times Q_{\varepsilon}(y, \eta \backslash y \cup x, \xi) \lambda(d \xi) d x \\
& -\sum_{x \in \eta} \int_{\mathbb{R}^{d}} \int_{\Gamma_{0}} a(x-y) k(\xi \cup \eta) \\
& \times Q_{\varepsilon}(y, \eta, \xi) \lambda(d \xi) d y
\end{aligned}
$$


where

$$
\begin{aligned}
Q_{\varepsilon}(y, \zeta, \xi):= & e\left(\tau_{y}^{(\varepsilon)}, \zeta\right) e\left(\varepsilon^{-1} t_{y}^{(\varepsilon)}, \xi\right)-e(-\phi(y-\cdot), \xi) \\
= & e\left(\varepsilon^{-1} t_{y}^{(\varepsilon)}, \xi\right)-e(-\phi(y-\cdot) \\
& -\left[1-e\left(\tau_{y}^{(\varepsilon)}, \zeta\right)\right] e\left(\varepsilon^{-1} t_{y}^{(\varepsilon)}, \xi\right) .
\end{aligned}
$$

For $t>0$, the function $e^{-t}-1+t$ takes positive values only; hence,

$$
\Psi(t):=\left(e^{-t}-1+t\right) / t^{2}, \quad t>0,
$$

is positive and bounded, say by $C>0$. Then by means of the inequality

$$
b_{1} \cdots b_{n}-a_{1} \cdots a_{n} \leq \sum_{i=1}^{n}\left(b_{i}-a_{i}\right) b_{1} \cdots b_{i-1} b_{i+1} \cdots b_{n}, \quad b_{i} \geq a_{1}>0
$$

we obtain

$$
\begin{aligned}
\left|e\left(\varepsilon^{-1} t_{y}^{(\varepsilon)}, \xi\right)-e(-\phi(y-), \xi)\right| \leq & \sum_{z \in \xi} \varepsilon[\phi(y-z)]^{2} \Psi(\varepsilon \phi(y-z)) \\
& \times \prod_{u \in \xi \backslash z} \phi(y-u) \\
\leq & \varepsilon C \sum_{z \in \xi}[\phi(y-z)]^{2} e(\phi(y-\cdot), \xi \backslash z),
\end{aligned}
$$

and

$$
\left|\left[1-e\left(\tau_{y}^{(\varepsilon)}, \zeta\right)\right] e\left(\varepsilon^{-1} t_{y}^{(\varepsilon)}, \xi\right)\right| \leq \varepsilon \sum_{z \in \zeta} \phi(y-z) e(\phi(y-\cdot), \xi) .
$$

Then from (4.24) for $\lambda$-almost all $\eta$ we have, see (3.8),

$$
\left|\left(L_{\varepsilon, \text { ren }}-L_{V}\right) k(\eta)\right| \leq \varepsilon\|k\|_{\vartheta_{0}}\left(\widetilde{C}|\eta| e^{-\vartheta_{0}|\eta|}+D(\eta) e^{-\vartheta_{0}|\eta|}\right),
$$

with

$$
\widetilde{C}=2 C \alpha\|\phi\|_{L^{\infty}\left(\mathbb{R}^{d}\right)}\langle\phi\rangle e^{-\vartheta_{0}}
$$

and

$$
D(\eta)=2 \alpha \exp \left(\langle\phi\rangle e^{-\vartheta_{0}}\right)\|\phi\|_{L^{\infty}\left(\mathbb{R}^{d}\right)}|\eta|(|\eta|+1) .
$$

Thus, we conclude that the expression in (.) in the right-hand side of (4.25) is in $\mathcal{K}_{\vartheta}$, which yields (4.23) and hence completes the proof.

Acknowledgments The authors are cordially grateful to the referee whose valuable and kind remarks were very helpful. This work was financially supported by the DFG through SFB 701: "Spektrale Strukturen und Topologische Methoden in der Mathematik", the IGK "Stochastics and Real World Models" and through the research project 436 POL 125/0-1, which is acknowledged by the authors.

Open Access This article is distributed under the terms of the Creative Commons Attribution License which permits any use, distribution, and reproduction in any medium, provided the original author(s) and the source are credited. 


\section{Appendix Proof of (3.54)}

For fixed $t \in(0, T(\vartheta))$ and $G_{0} \in B_{\mathrm{bs}}\left(\Gamma_{0}\right)$, by (3.24) we have

$$
\left\langle\left\langle G_{0}, k_{t}\right\rangle\right\rangle-\left\langle\left\langle G_{0}, k_{t}^{\Lambda_{n}, N_{l}}\right\rangle=\left\langle\left\langle G_{t}, k_{0}\right\rangle\right\rangle-\left\langle\left\langle G_{t}, q_{0}^{\Lambda_{n}, N_{l}}\right\rangle=: \mathcal{I}_{n}^{(1)}+\mathcal{I}_{n, l}^{(2)},\right.\right.
$$

where we set

$$
\begin{aligned}
& \mathcal{I}_{n}^{(1)}=\int_{\Gamma_{0}} G_{t}(\eta) k_{0}(\eta)\left(1-\mathbb{I}_{\Gamma_{\Lambda_{n}}}(\eta)\right) \lambda(d \eta), \\
& \mathcal{I}_{n, l}^{(2)}=\int_{\Gamma_{0}} G_{t}(\eta)\left[k_{0}(\eta) \mathbb{I}_{\Gamma_{\Lambda_{n}}}(\eta)-q_{0}^{\Lambda_{n}, N_{l}}(\eta)\right] \lambda(d \eta) .
\end{aligned}
$$

Let us prove that, for an arbitrary $\varepsilon>0$,

$$
\left|\mathcal{I}_{n}^{(1)}\right|<\varepsilon / 2,
$$

for sufficiently big $\Lambda_{n}$. Recall that $k_{0}$ is a correlation function, and hence is positive. Taking into account that

$$
\mathbb{I}_{\Gamma_{\Lambda}}(\eta)=\prod_{x \in \eta} \mathbb{I}_{\Lambda}(x)
$$

we write

$$
\begin{aligned}
\left|\mathcal{I}_{n}^{(1)}\right| \leq & \int_{\Gamma_{0}}\left|G_{t}(\eta)\right| k_{0}(\eta)\left(1-\mathbb{I}_{\Gamma_{\Lambda_{n}}}(\eta)\right) \lambda(d \eta) \\
= & \sum_{p=1}^{\infty} \frac{1}{p !} \int_{\left(\mathbb{R}^{d}\right)^{p}}\left|\left(G_{t}\right)^{(p)}\left(x_{1}, \ldots x_{p}\right)\right| k_{0}^{(p)}\left(x_{1}, \ldots x_{p}\right) \\
& \times J_{\Lambda_{n}}\left(x_{1}, \ldots x_{p}\right) d x_{1} \ldots d x_{p}
\end{aligned}
$$

where

$$
\begin{aligned}
J_{\Lambda}\left(x_{1}, \ldots, x_{p}\right): & =1-\mathbb{I}_{\Lambda}\left(x_{1}\right) \cdots \mathbb{I}_{\Lambda}\left(x_{p}\right) \\
:= & \mathbb{I}_{\Lambda^{c}}\left(x_{1}\right) \mathbb{I}_{\Lambda}\left(x_{2}\right) \cdots \mathbb{I}_{\Lambda}\left(x_{p}\right)+\mathbb{I}_{\Lambda^{c}}\left(x_{2}\right) \mathbb{I}_{\Lambda}\left(x_{3}\right) \cdots \mathbb{I}_{\Lambda}\left(x_{p}\right) \\
& +\cdots+\mathbb{I}_{\Lambda^{c}}\left(x_{p-1}\right) \mathbb{I}_{\Lambda}\left(x_{p}\right)+\mathbb{I}_{\Lambda^{c}}\left(x_{p}\right), \\
\leq & \sum_{s=1}^{p} \mathbb{I}_{\Lambda^{c}}\left(x_{s}\right),
\end{aligned}
$$

and $\Lambda^{c}:=\mathbb{R}^{d} \backslash \Lambda$. Taking into account that $k_{0}=k_{\mu_{0}} \in \mathcal{K}_{\vartheta_{0}}$, by (5.4) we obtain in (5.3)

$$
\left|\mathcal{I}_{n}^{(1)}\right| \leq\left\|k_{0}\right\|_{\alpha^{*}} \sum_{p=1}^{\infty} \frac{p}{p !} e^{-\alpha^{*} p} \int_{\Lambda_{n}^{c}} \int_{\left.\mathbb{R}^{d}\right)^{p-1}}\left|\left(G_{t}\right)^{(p)}\left(x_{1}, \ldots x_{p}\right)\right| d x_{1} \cdots d x_{p} .
$$


For $t$ as in (5.1), one finds $\vartheta<\vartheta_{0}$ such that $G_{t} \in \mathcal{G}_{\vartheta}$, cf. Theorem 3.2. For this $\vartheta$ and $\varepsilon$ as in (5.2), we pick $\bar{p} \in \mathbb{N}$ such that

$$
\sum_{p=\bar{p}+1}^{\infty} \frac{e^{-\vartheta p}}{p !} \int_{\left(\mathbb{R}^{d}\right)^{p}}\left|\left(G_{t}\right)^{(p)}\left(x_{1}, \ldots x_{p}\right)\right| d x_{1} \cdots d x_{p}<\frac{\varepsilon e\left(\vartheta_{0}-\vartheta\right)}{4\left\|k_{0}\right\|_{\vartheta_{0}}} .
$$

Then we apply (5.6) and the following evident estimate

$$
p e^{-\vartheta_{0} p} \leq e^{-\vartheta p} / e\left(\vartheta_{0}-\vartheta\right)
$$

and obtain in (5.5) the following

$$
\begin{aligned}
\left|\mathcal{I}_{n}^{(1)}\right| & <\frac{\left\|k_{0}\right\|_{\vartheta_{0}}}{e\left(\vartheta_{0}-\vartheta\right)} \sum_{p=1}^{\bar{p}} \frac{p}{p !} e^{-\vartheta_{0} p} \int_{\Lambda_{n}^{c}} \int_{\left(\mathbb{R}^{d}\right)^{p-1}}\left|\left(G_{t}\right)^{(p)}\left(x_{1}, \ldots x_{p}\right)\right| d x_{1} \cdots d x_{p} \\
& +\varepsilon / 4 .
\end{aligned}
$$

Here the first term contains a finite number of summands, in each of which $\left(G_{t}\right)^{(p)}$ is in $L^{1}\left(\left(\mathbb{R}^{d}\right)^{p}\right)$. Hence, it can be made strictly smaller than $\varepsilon / 4$ by picking big enough $\Lambda_{n}$, which yields (5.2).

Let us show the same for the second integral in (5.1). Write, see (3.37), (3.43), and (3.39),

$$
\begin{aligned}
\mathcal{I}_{n, l}^{(2)} & =\int_{\Gamma_{0}} G_{t}(\eta) \int_{\Gamma_{0}} R_{0}^{\Lambda_{n}}(\eta \cup \xi)\left[1-I_{N_{l}}(\eta \cup \xi)\right] \lambda(d \eta) \lambda(d \xi) \\
& =\int_{\Gamma_{0}} F_{t}(\eta) R_{0}^{\Lambda_{n}}(\eta)\left[1-I_{N_{l}}(\eta)\right] \lambda(d \eta) \\
& =\sum_{m=N_{l}+1}^{\infty} \frac{1}{m !} \int_{\Lambda_{n}^{m}}\left(R_{0}^{\Lambda_{n}}\right)^{(m)}\left(x_{1}, \ldots, x_{m}\right) \\
& \times F_{t}^{(m)}\left(x_{1}, \ldots, x_{m}\right) d x_{1} \cdots d x_{m},
\end{aligned}
$$

where

$$
F_{t}(\eta):=\left(K G_{t}\right)(\eta)=\sum_{\xi \subset \eta} G_{t}(\xi)
$$

and hence

$$
F_{t}^{(m)}\left(x_{1}, \ldots, x_{m}\right)=\sum_{s=0}^{m} \sum_{\left\{i_{1}, \ldots, i_{s}\right\} \subset\{1, \ldots, m\}}\left(G_{t}\right)^{(s)}\left(x_{i_{1}}, \ldots, x_{i_{s}}\right) .
$$

By (3.37), for $x_{i} \in \Lambda_{n}, i=1, \ldots, m$, we have

$$
k_{0}^{(m)}\left(x_{1}, \ldots, x_{m}\right)=\sum_{s=0}^{\infty} \int_{\Lambda_{n}^{s}}\left(R_{0}^{\Lambda_{n}}\right)^{(m+s)}\left(x_{1}, \ldots, x_{m}, y_{1}, \ldots y_{s}\right) d y_{1} \cdots d y_{s},
$$

from which we immediately get that

$$
\left(R_{0}^{\Lambda_{n}}\right)^{(m)}\left(x_{1}, \ldots, x_{m}\right) \leq k_{0}^{(m)}\left(x_{1}, \ldots, x_{m}\right) \leq e^{-\vartheta_{0} m}\left\|k_{0}\right\|_{\vartheta_{0}},
$$


since $k_{0} \in \mathcal{K}_{\vartheta_{0}}$. Now let $\Lambda_{n}$ be such that (5.2) holds. Then we can have

$$
\left|\mathcal{I}_{n, l}^{(2)}\right|<\varepsilon / 2
$$

holding for big enough $N_{l}$ if $e^{-\vartheta_{0}|\cdot|} F_{t}$ is in $L^{1}\left(\Lambda_{n}, d \lambda\right)$. By (5.7),

$$
\begin{aligned}
& \sum_{p=0}^{\infty} \frac{1}{p !} e^{-\vartheta_{0} p} \int_{\Lambda_{n}^{p}}\left|F^{(p)}\left(x_{1}, \ldots, x_{p}\right)\right| d x_{1} \cdots d x_{p} \\
& \quad \leq \sum_{p=0}^{\infty} \sum_{s=0}^{p} \frac{1}{s !(p-s) !} e^{-\vartheta_{0} s}\left\|\left(G_{t}\right)^{(s)}\right\|_{L^{1}\left(\Gamma_{0}, d \lambda\right)} e^{-\vartheta_{0}(p-s)}\left[m\left(\Lambda_{n}\right)\right]^{p-s} \\
& \quad=\left\|G_{t}\right\|_{\vartheta_{0}} \exp \left(e^{-\vartheta_{0}} m\left(\Lambda_{n}\right)\right)
\end{aligned}
$$

where $m\left(\Lambda_{n}\right)$ is the Lebesgue measure of $\Lambda_{n}$, cf. (2.8). This yields (5.8) and thereby also (3.54).

\section{References}

1. Albeverio, S.: Kondratiev, YuG, Röckner, M.: Analysis and geometry on configuration spaces. J. Funct. Anal. 154, 444-500 (1998)

2. Bogoliubov, N.N.: Problems of Dynamical Theory in Statistical Physics. Gosudarstv. Izdat. Tehn.-Teor. Lit., Moscow-Leningrad (1946)

3. Bovier, A., den Hollander, F., Spitoni, C.: Homogeneous nucleation for Glauber and Kawasaki dynamics in large volumes at low temperatures. Ann. Probab. 38, 661-713 (2010)

4. Caglioti, E., Marchioro, C., Pulvirenti, M.: Non-equilibrium dynamics of three-dimensional infinite particle systems. Commun. Math. Phys. 215, 25-43 (2000)

5. Cox, J.T.: Coalescenting random walk and voter model consensus times on the torus in $\mathbb{Z}^{d}$. Ann. Probab. 17, 1333-1366 (1989)

6. Deimling, K.: Ordinary Differential Equations in Banach Spaces. In: Lecture Notes in Mathematics, vol. 596, Springer, Berlin (1977)

7. Dobrushin, R.L., Sinai, Y.G., Sukhov, Y.M.: Dynamical systems of statistical mechanics. In: Sinai, Y.G. (ed.) Ergodic Theory with Applications to Dynamical Systems and Statistical Mechanics, vol. II. Encyclopaedia Mathematical Science. Springer, Berlin (1989)

8. Gaudillière, A., den Hollander, F., Nardia, F.R., Olivieri, E., Scoppola, E.: Ideal gas approximation for a two-dimensional rarefied gas under Kawasaki dynamics. Stochastic Process. Appl. 119, 737-774 (2009)

9. Finkelshtein, D.L., Kondratiev, Y.G., Kozitsky, Y.: Glauber Dynamics in Continuum: A Constructive Approach to Evolution of States. Preprinf 11034, SFB 701. Universität Bielefeld, Bielefeld (to appear in, Discrete Continuous Dynamics Systems A (2011)

10. Finkelshtein, D.L., Kondratiev, Y.G., Kozitsky, Y., Kutoviy, O.: Markov Dynamics in a Spatial Ecological Model with Dispersion and Competition. Preprinf 11034, SFB 701. Universität Bielefeld, Bielefeld (2011)

11. Finkelshtein, D.L., Kondratiev, Y.G., Kutovyi, O.: Semigroup approach to birth-and-death stochastic dynamics in continuum. J. Funct. Anal. 262, 1274-1308 (2012)

12. Finkelshtein, D.L., Kondratiev, Y.G., Kutovyi, O.: Vlasov scaling for stochastic dynamics of contiuous systems. J. Stat. Phys. 141, 158-178 (2010)

13. Finkelshtein, D.L., Kondratiev, Y.G., Kutovyi, O.: Individual based model with competition in spatial ecology. SIAM J. Math. Anal. 41(1), 297-317 (2009)

14. Finkelshtein, D.L., Kondratiev, Y.G., Lytvynov, E.W.: Equilibrium Glauber dynamics of continuous particle systems as a scaling limit of Kawasaki dynamics. Random Oper. Stoch. Equ. 15, 105-126 (2007)

15. Finkelshtein, D.L., Kondratiev, Y.G., Oliveira, M.J.: Markov evolution and hierarchical equations in the continuum. I: one-component systems. J. Evol. Equ. 9, 197-233 (2009)

16. Fritz, J., Dobrushin, R.L.: Non-equilibrium dynamics of two-dimensional infinite particle systems with a singular interaction. Comm. Math. Phys. 57, 67-81 (1977)

17. Kawasaki, K.: Diffusion constants near the critical point for time-dependent Ising models. Phys. Rev. 145, 224-230 (1966) 
18. Kawasaki, K.: Kinetics of Ising models. In: Domb, C., Green, M.S. (eds.) Phase Transitions and Critical Phenomena, vol. 2, pp. 443-501. Academic Press, London (1972)

19. Kondratiev, Y.G., Kuna, T.: Harmonic analysis on configuration space. I. General theory. Infin. Dimens. Anal. Quantum Probab. Relat. Top. 5, 201-233 (2002)

20. Kondratiev, Y.G., Kutovyi, O.: On the metrical properties of the configuration space. Math. Nachr. 279, 774-783 (2006)

21. Kondratiev, Y.G., Lytvynov, E., Röckner, M.: Equilibrium Kawasaki dynamics of continuous particle systems. Infin. Dimens. Anal. Quantum Probab. Relat. Top. 10, 185-209 (2007)

22. Kondratiev, Y.G., Lytvynov, E., Röckner, M.: Non-equilibrium stochastic dynamics in continuum: the free case. Cond. Matt. Phys. 11, 701-721 (2008)

23. Lanford III, O.E.: III The classical mechanics of one-dimensional systems of infinitely many particles. I. An existence theorem. Commun. Math. Phys. 9, 176-191 (1968)

24. Liggett T.M.: Interacting Particle Systems. In: Grundlehren der Mathematischen Wissenschaften, vol. 276. Springer, New York (1985)

25. Liggett, T.M.: Stochastic models for large interacting systems and related correlation inequalities. Proc. Natl. Acad. Sci. USA 107, 16413-16419 (2010)

26. Neuhauser, C.: Mathematical challenges in spatial ecology. Notices of AMS 48, 1304-1314 (2001)

27. Obata, N.: Configuration space and unitary representations of the group of diffeomorphisms. RIMS Kôkyûroku 615, 129-153 (1987)

28. Pazy, A.: Semigroups of Linear Operators and Applications to Partial Differential Equations. In: Applied Mathematical Sciences, vol. 44. Springer, New York (1983)

29. Presutti, E.: Scaling Limits in Statistical Mechanics and Microstructures in Continuum Mechanics. In: Theoretical and Mathematical Physics. Springer, Berlin (2009)

30. Ruelle, D.: Statistical Mechanics: Rigorous Results. World Scientific, Singapore (1999)

31. Spohn, H.: Large Scale Dynamics of Interacting Particles. In: Texts and Monographs in Physics. Springer, Berlin (1991)

32. Thieme, H.R., Voigt, J.: Stochastic semigroups: their construction by perturbation and approximation. In: Positivity IV-Theory and Applications, pp. 135-146. Technical University of Dresden, Dresden (2006)

33. Trèves, F. (1968). Ovcyannikov Theorem and Hyperdifferential Operators. In: Notas de Matemática, vol. 46. Instituto de Matemática Pura e Aplicada, Conselho Nacional de Pesquisas, Rio de Janeiro 